Gazi University
Journal of Science
$\mathrm{http} / /$ dergipark.gov.tr/gujs

\title{
A Comparative Analysis of the Ranking Functions for the IVIFVs and A New Score Function
}

\author{
Melda KOKOC ${ }^{1, *}$, Suleyman ERSOZ ${ }^{2}$ (D) \\ ${ }^{I}$ Gazi University, Rectorate, 06560, Ankara, Turkey \\ ${ }^{2}$ Kirlkkale University, Department of Industrial Engineering, 71451, Kırlkkale, Turkey
}

\section{Highlights}

- A new score function of IVIFVs is introduced.

- The limitations of the existing score and accuracy functions are determined.

- A comparative analysis for the ranking functions of the IVIFVs is performed.

\section{Article Info}

Received: 16 Dec 2020

Accepted: 08 Dec 2021

\section{Keywords}

Accuracy function,

Interval-valued

intuitionistic fuzzy set,

Ranking,

Score function

\begin{abstract}
The ranking of interval-valued intuitionistic fuzzy values (IVIFVs) has an important role in reallife decision-making problems. Even though there are many approaches related to the ranking methods of the IVIFVs, some of them have some shortcomings. In this study, the disadvantages of the existing ranking functions of the IVIFVs are discussed. It is revealed that both the most popular ranking functions and their recently improved versions may lead to unacceptable results. Furthermore, in this article, a new score function is offered to cope with the shortcomings of the ranking functions. The importance and performance of this new score function are proven with the help of many examples. Also, the decision-making algorithm adapted by integrating the new function is presented to illustrate the applicability of the new score function in the decisionmaking problems.
\end{abstract}

\section{INTRODUCTION}

In case there is uncertainty and lack of information, it is difficult to evaluate alternatives correctly by considering several criteria. How to cope with vagueness and ambiguity is an interesting and significant issue [1]. To deal with vagueness and ambiguity Zadeh [2] offered fuzzy sets (FSs) theory. In the fuzzy set theory, the belonging of the element to the set is represented by the membership degree. Atanassov [3] put forward that the belonging of the element to the set should be defined by the membership, non-membership, and hesitant degrees instead of just its membership degree. So, he suggested the intuitionistic fuzzy sets (IFSs) theory as an extension of fuzzy sets. IFSs theory is more flexible and effective than traditional FSs theory in dealing with the vagueness and ambiguity of the objectives [4-7]. But, in some complex decisionmaking situations, decision-makers may not have adequate information to assign crisp values to membership and non-membership degrees. Hence, Atanassov and Gargov [8] proposed the interval-valued intuitionistic fuzzy sets (IVIFSs) theory in which the membership degree and non-membership degree of an element are described with closed intervals instead of crisp values. Owing to the fact that the membership degree and the non-membership degree of an element are defined by intervals, researchers have considered IVIFSs as a suitable and realistic tool to express ambiguous and vague information in real-life problems [4, $5,9-11]$. One of these real-life problems is the multi-criteria decision-making (MCDM) problem. The main goal in solving MCDM problems is to rank the alternatives by taking into consideration criteria. But, when the evaluate the alternatives in the IVIF environment, the problem of how to compare the IVIF values (IVIFVs) is revealed. So, the ranking of the IVIFVs is an attention-grabbing topic. 
The problem of ranking IVIFVs, though, has been extensively studied, there is an agreed conclusion that there is no unique best approach to do this. Therefore, it is important to develop the single-stage ranking function that offers the best possible performance. Several approaches have been developed for ranking IVIFVs. Among these, score functions [6, 9, 10, 12-19] and accuracy functions [5, 7, 12, 15, 17, 18, 20-25] are frequently preferred approaches. However, in some cases, the existing score or accuracy functions for ranking IVIFVs do not yield sufficient performance and they cannot distinguish comparable IVIFVs. Therefore, there is a need for an improved score function that will handle the shortcomings of the existing score functions and give sufficient information about the IVIFVs. With this motivation, in this study, a new score function, which can rank or distinguish two IVIFVs more efficiently than the existing functions, is developed. This study stands out as the most comprehensive study comparing existing score functions and accuracy functions, in addition to proposing a high-performance score function.

This paper is organized as follows. The basic concepts of interval-valued intuitionistic fuzzy sets and monotonicity property of score function and of accuracy function are given in Section 2. In Section 3, existing ranking functions are presented and their shortcomings are proven with examples. The proposed score function is introduced in Section 4. A comparative performance analysis is performed with twenty IVIFV pairs to show that the proposed function is more reasonable in the ranking of IVIFVs in Section 5. In Section 6, Şahin [23]'s decision-making algorithm is adapted to show the application of the new score function in MCDM problems. Conclusions are presented in the last section.

\section{BASIC CONCEPTS}

Some basic definitions of IVIFSs are presented in this section.

Definition 1.[8] Let IVIFS(X) denotes the family of all the IVIFSs over the universe of discourse $X=\left\{x_{1}, x_{2}, \ldots, x_{n}\right\}$ and $\tilde{A} \in \operatorname{IVIFS}(X)$ be an IVIFV given by $\tilde{A}=\left\langle\left[\mu_{\tilde{A}}^{L}(x), \mu_{\tilde{A}}^{U}(x)\right],\left[v_{\tilde{A}}^{L}(x), v_{\tilde{A}}^{U}(x)\right]\right\rangle$.

Definition 2. [8] Let $\tilde{A}$ be an IVIFV. The hesitant degree of $\tilde{A}$ is defined as follows $\pi_{\tilde{A}}=\left[\pi_{\tilde{A}}^{L}(x), \pi_{\tilde{A}}^{U}(x)\right]=\left\langle\left[1-\mu_{\tilde{A}}^{U}(x)-v_{\tilde{A}}^{U}(x)\right],\left[1-\mu_{\tilde{A}}^{L}(x)-v_{\tilde{A}}^{L}(x)\right]\right\rangle$.

Definition 3. [8] Let $\tilde{A}, \tilde{B} \in \operatorname{IVIFS}(X)$. A subset relation $\tilde{A} \subset \tilde{B} \Leftrightarrow \mu_{\tilde{A}}^{L}(x) \leq \mu_{\tilde{B}}^{L}(x), \mu_{\tilde{A}}^{U}(x) \leq \mu_{\tilde{B}}^{U}(x)$, $v_{\tilde{A}}^{L}(x) \geq v_{\tilde{B}}^{L}(x)$ and $v_{\tilde{A}}^{U}(x) \geq v_{\tilde{B}}^{U}(x), \forall x \in X$. In addition, $\tilde{A}=\tilde{B} \Leftrightarrow \tilde{A} \subset \tilde{B}$ and $\tilde{A} \supset \tilde{B}$.

Definition 4. [12] Let $\tilde{A}_{j}$ for $j=1,2, \ldots, n$ is a collection of IVIFVs. The IVIF weighted arithmetic (IVIFWA) operator is defined as:

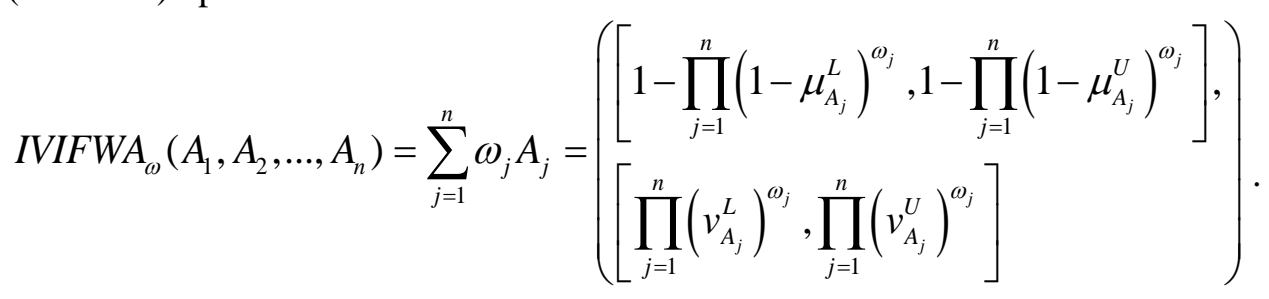

Definition 5. [18] Let $\tilde{A}$ be an IVIFV. The monotonicity property for the score function $S(\tilde{A})$ of the $\tilde{A}$ as below.

1) If $b, c, d$ are fixed, then $\partial S(\tilde{A}) / \partial a>0$,

2) If $a, c, d$ are fixed, then $\partial S(\tilde{A}) / \partial b>0$,

3) If a, $b, d$ are fixed, then $\partial S(\tilde{A}) / \partial c<0$,

4) If a, $b, c$ are fixed, then $\partial S(\tilde{A}) / \partial d<0$. 
Definition 6. [18] Let $\tilde{A}$ be an IVIFV. The monotonicity property for the accuracy function $A(\tilde{A})$ of the $\tilde{A}$ as below.

1) If $b, c, d$ are fixed, then $\partial A(\tilde{A}) / \partial a>0$,

2) If $a, c, d$ are fixed, then $\partial A(\tilde{A}) / \partial b>0$,

3) If a, $b, d$ are fixed, then $\partial A(\tilde{A}) / \partial c>0$,

4) If a, $b, c$ are fixed, then $\partial A(\tilde{A}) / \partial d>0$.

\section{EXISTING RANKING FUNCTIONS}

The basic principle of ranking functions is that the greater score value or accuracy value of $\tilde{A}$, the greater IVIFV $\tilde{A}$. In the light of this principle, it is determined that the existing score and accuracy functions have counter-intuitive cases on the ranking of IVIFVs. So, these functions' capacity cannot be enough to distinguish some IVIFV pairs. These counter-intuitive cases are illustrated with examples under the related functions' titles.

\subsection{Xu[12]'s Ranking Method of the IVIFV}

To rank the IVIFVs, $\mathrm{Xu}[12]$ developed the score function $S($.$) and the accuracy function H($.$) as follows:$

$$
\begin{aligned}
& S(\tilde{A})=\frac{\mu_{\tilde{A}}^{L}-v_{\tilde{A}}^{L}+\mu_{\tilde{A}}^{U}-v_{\tilde{A}}^{U}}{2}, \\
& H(\tilde{A})=\frac{\mu_{\tilde{A}}^{L}+\mu_{\tilde{A}}^{U}+v_{\tilde{A}}^{L}+v_{\tilde{A}}^{U}}{2} .
\end{aligned}
$$

Example 1. Let $\tilde{A}_{1}=[0.65,0.70],[0.03,0.10]$ and $\tilde{A}_{2}=[0.57,0.78],[0.06,0.07]$ be two IVIFVs. It is obtained that $S\left(\tilde{A}_{1}\right)=S\left(\tilde{A}_{2}\right)=0.61$ and $H\left(\tilde{A}_{1}\right)=H\left(\tilde{A}_{2}\right)=0.74$ by using Equations (1) and (2). Thus, there is a ranking problem with $\mathrm{Xu}$ [12]'s score function and accuracy function because of the inadequate use of the upper and lower bounds of the membership and non-membership.

\subsection{Lee's [13] Ranking Method of the IVIFV}

Due to the fact that Xu's [12] ranking techniques cannot give sufficient information in some cases, Lee [13] offered a new score function $S($.

$$
S(\tilde{A})=\frac{2+\mu_{\tilde{A}}^{L}+\mu_{\tilde{A}}^{U}-v_{\tilde{A}}^{L}-v_{\tilde{A}}^{U}}{3-\mu_{\tilde{A}}^{L}-\mu_{\tilde{A}}^{U}-v_{\tilde{A}}^{L}-v_{\tilde{A}}^{U}} .
$$

Example 2. Let $\tilde{A}_{1}=[0.07,0.17],[0.27,0.37]$ and $\tilde{A}_{2}=[0.10,0.17],[0.12,0.52]$ be two IVIFVs. Using Equation (3), score values are obtained as $S\left(\tilde{A}_{1}\right)=S\left(\tilde{A}_{2}\right)=0.75$, and so, Lee's [13] score function is inadequate to rank.

\subsection{Ye's [5] Ranking Method of the IVIFV}

Ye [5] proposed a new accuracy function by considering the hesitant degree of IVIFVs, and he put forward that this function is more reasonable than the accuracy function developed by $\mathrm{Xu}$ 


$$
M(\tilde{A})=\mu_{\tilde{A}}^{L}+\mu_{\tilde{A}}^{U}-1+\frac{v_{\tilde{A}}^{L}+v_{\tilde{A}}^{U}}{2} .
$$

Example 3. Let that $\tilde{A}_{1}=[0.50,0.60],[0.20,0.20]$ and $\tilde{A}_{2}=[0.40,0.70],[0.10,0.30]$ be two IVIFVs. Using Equation (4), accuracy values are obtained as $M\left(\tilde{A}_{1}\right)=M\left(\tilde{A}_{2}\right)=0.30$, and so, Ye's [5] accuracy function cannot distinguish these two IVIFVs.

\subsection{Nayagam et al.'s [20] Ranking Method of the IVIFV}

Nayagam et al. [20] offered an accuracy function $L($.$) that can provide a useful way to help to make a$ decision

$$
L(\tilde{A})=\frac{\mu_{\tilde{A}}^{L}+\mu_{\tilde{A}}^{U}-v_{\tilde{A}}^{U}\left(1-\mu_{\tilde{A}}^{U}\right)-v_{\tilde{A}}^{L}\left(1-\mu_{\tilde{A}}^{L}\right)}{2} .
$$

Example 4. Let $\tilde{A}_{1}=[0.55,0.67],[0.12,0.24]$ and $\tilde{A}_{2}=[0.58,0.68],[0.16,0.28]$ be two IVIFVs and $\tilde{A}_{1} \supset \tilde{A}_{2}$. By applying $L(\tilde{A})$ presented in Equation (5), accuracy values are calculated as $L\left(\tilde{A}_{1}\right)=0.54$ and $L\left(\tilde{A}_{2}\right)=0.55$. That is, the rank obtained by Nayagam et al.'s [20] accuracy function is the reverse of the expected $\operatorname{rank} \tilde{A}_{1}>\tilde{A}_{2}$.

\subsection{Nayagam and Sivaraman [21]'s Ranking Method of the IVIFV}

A new general accuracy function $L G($.$) was presented by Nayagam and Sivaraman [21] to defeat that$ ranking functions arise illogical results where $0 \leq \delta \leq 1$

$$
L G(\tilde{A})=\frac{\mu_{\tilde{A}}^{L}+\mu_{\tilde{A}}^{U}+\delta\left(2-\mu_{\tilde{A}}^{L}-\mu_{\tilde{A}}^{U}-v_{\tilde{A}}^{L}-v_{\tilde{A}}^{U}\right)}{2} .
$$

Example 5. Let $\tilde{A}_{1}=[0.58,0.70],[0.28,0.30]$ and $\tilde{A}_{2}=[0.35,0.50],[0.05,0.10]$ be two IVIFVs. Using Equation (6), when $\delta=0.5$, accuracy values are calculated as $L G\left(\tilde{A}_{1}\right)=L G\left(\tilde{A}_{2}\right)=0.57$, and so, Nayagam and Sivaraman [21]'s score function has drawback.

\subsection{Tu and Chen [6]'s Ranking Method of the IVIFV}

Tu and Chen [6] proposed two score functions based on the opinion that the minimum of membership and the maximum of non-membership showed the least prioritized ranking

$$
\begin{aligned}
& D_{o}(\tilde{A})=\mu_{\tilde{A}}^{L}\left(2-\mu_{\tilde{A}}^{L}-2 v_{\tilde{A}}^{U}\right), \\
& D_{p}(\tilde{A})=1-v_{\tilde{A}}^{L}\left(2-v_{\tilde{A}}^{L}-2 \mu_{\tilde{A}}^{U}\right) .
\end{aligned}
$$

Example 6. Let $\tilde{A}_{1}=[0.10,0.25],[0.38,0.45]$ and $\tilde{A}_{1}=[0.10,0.45],[0.42,0.45]$ be two IVIFVs. By applying $D_{o}(\tilde{A})$ presented in Equation (7), score values are obtained as $D_{o}\left(\tilde{A}_{1}\right)=D_{o}\left(\tilde{A}_{2}\right)=0.10$. That is, $D_{o}(\tilde{A})$ produces equal score value when $\mu_{\tilde{A}_{1}}^{L}=\mu_{\tilde{A}_{2}}^{L}$ and $v_{\tilde{A}_{1}}^{U}=v_{\tilde{A}_{2}}^{U}$ without affecting $\mu_{\tilde{A}}^{U}$ and $v_{\tilde{A}}^{L}$, and it cannot separate IVIFVs. 
Example 7. Let $\tilde{A}_{1}=[0.16,0.50],[0.25,0.29]$ and $\tilde{A}_{2}=[0.37,0.50],[0.25,0.36]$ be two IVIFVs. By applying $D_{p}(\tilde{A})$ presented in Equation (8), score values are obtained as $D_{p}\left(\tilde{A}_{1}\right)=D_{p}\left(\tilde{A}_{2}\right)=0.813$. That is, $D_{p}(\tilde{A})$ produces equal score value when $\mu_{\tilde{A}_{1}}^{U}=\mu_{\tilde{A}_{2}}^{U}$ and $v_{\tilde{A}_{1}}^{L}=v_{\tilde{A}_{2}}^{L}$ without affecting $\mu_{\tilde{A}}^{L}$ and $v_{\tilde{A}}^{U}$, and it cannot distinguish IVIFVs.

\subsection{Bai [14]'s Ranking Method of the IVIFV}

Bai [14] introduced an improved score function $I($.$) for the ranking order of IVIFVs$

$$
I(\tilde{A})=\frac{\mu_{\tilde{A}}^{L}+\mu_{\tilde{A}}^{L}\left(1-\mu_{\tilde{A}}^{L}-v_{\tilde{A}}^{L}\right)+\mu_{\tilde{A}}^{U}+\mu_{\tilde{A}}^{U}\left(1-\mu_{\tilde{A}}^{U}-v_{\tilde{A}}^{U}\right)}{2} .
$$

Example 8. If corresponding to two different IVIFVs $\tilde{A}_{1}=[0.094,0.262],[0.439,0.687], \tilde{A}_{2}=[0.088,0.27]$, $[0.437,0.683]$ and $\tilde{A}_{1} \subset \tilde{A}_{2}$. Using Equation (9), score values are obtained as $I\left(\tilde{A}_{1}\right)=0.2062$ and $I\left(\tilde{A}_{2}\right)=0.2058$, and so, the Bai (2013)'s score function $I(\tilde{A})$ fails to rank these two IVIFVs.

\subsection{Wang and Niu [15]'s Ranking Method of the IVIFV}

Wang and Niu [15] developed the score function $W_{S}($.$) and the accuracy function W_{H}($.$) to overcome the$ disadvantages of existing functions

$$
\begin{aligned}
& W_{S}(\tilde{A})=\left(\frac{\mu_{\tilde{A}}^{L}+\mu_{\tilde{A}}^{U}}{2}-\frac{v_{\tilde{A}}^{L}+v_{\tilde{A}}^{U}}{2}\right)\left(1+\frac{\left(1-\mu_{\tilde{A}}^{U}-v_{\tilde{A}}^{U}\right)+\left(1-\mu_{\tilde{A}}^{L}-v_{\tilde{A}}^{L}\right)}{2}\right), \\
& W_{H}(\tilde{A})=\left(\frac{\mu_{\tilde{\tilde{A}}}^{L}+\mu_{\tilde{A}}^{U}}{2}+\frac{v_{\tilde{A}}^{L}+v_{\tilde{A}}^{U}}{2}\right)\left(1+\frac{\left(1-\mu_{\tilde{A}}^{U}-v_{\tilde{A}}^{U}\right)+\left(1-\mu_{\tilde{A}}^{L}-v_{\tilde{A}}^{L}\right)}{2}\right) .
\end{aligned}
$$

Example 9. Let $\tilde{A}_{1}=[0.38,0.38],[0.20,0.40]$ and $\tilde{A}_{2}=[0.26,0.50],[0.15,0.45]$ be two IVIFVs. Using Equations (10) and (11), score values are obtained as $W_{S}\left(\tilde{A}_{1}\right)=W_{S}\left(\tilde{A}_{2}\right)=0.106$ and accuracy values are obtained as $W_{H}\left(\tilde{A}_{1}\right)=W_{H}\left(\tilde{A}_{2}\right)=0.898$. Score values and accuracy values shows that, Wang and Niu [15]'s score function and accuracy function cannot distinguish IVIFVs. Also, if the midpoints of the membership and the non-membership coincide, the score function $W_{S}($.$) will equal to 0$.

\subsection{Joshi and Kharayat [7]'s Ranking Method of the IVIFV}

Joshi and Kharayat [7] gave an accuracy function $P($.$) as follows$

$$
P(\tilde{A})=\frac{\mu_{\tilde{A}}^{L}+\mu_{\tilde{A}}^{U}+\mu_{\tilde{A}}^{L} \mu_{\tilde{A}}^{U}-v_{\tilde{A}}^{L} v_{\tilde{A}}^{U}}{3}
$$

Example 10. Let $\tilde{A}_{1}=[0.20,0.20],[0.36,0.42]$ and $\tilde{A}_{2}=[0.20,0.20],[0.24,0.63]$ be two IVIFVs. By applying $P\left(\right.$.) in Equation (12), accuracy values are obtained as $P\left(\tilde{A}_{1}\right)=P\left(\tilde{A}_{2}\right)=0.096$. Thus, this accuracy function fails to separate IVIFVs.

\subsection{Kang et al. [22]'s Ranking Method of the IVIFV}

A new accuracy function $H_{K}($.$) was defined by Kang et al. [22]$ 


$$
H_{K}(\tilde{A})=\frac{\mu_{\tilde{A}}^{L}-v_{\tilde{A}}^{L}+\mu_{\tilde{A}}^{U}-v_{\tilde{A}}^{U}-v_{\tilde{A}}^{L}\left(1-\mu_{\tilde{A}}^{U}-v_{\tilde{A}}^{U}\right)}{2}-\frac{v_{\tilde{A}}^{U}\left(1-\mu_{\tilde{A}}^{L}-v_{\tilde{A}}^{L}\right)}{2} .
$$

Example 11. Let $\tilde{A}_{1}=[0.13,0.41],[0.43,0.43]$ and $\tilde{A}_{2}=[0.22,0.32],[0.43,0.43]$ be two IVIFVs. By applying $H_{K}\left(\right.$.) in Equation (13), accuracy values are calculated as $H_{K}\left(\tilde{A}_{1}\right)=H_{K}\left(\tilde{A}_{2}\right)=-0.289$. In this case, ranking cannot be made because both values are equal to each other.

\subsection{Garg [9]'s Ranking Method of the IVIFV}

Garg [9] presented a new generalized improved score function GIS(.) by considering the weighted average of the degree of hesitation

$$
\operatorname{GIS}(\tilde{A})=\frac{\mu_{\tilde{A}}^{L}+\mu_{\tilde{A}}^{U}}{2}+k_{1} \mu_{\tilde{A}}^{L}\left(1-\mu_{\tilde{A}}^{L}-v_{\tilde{A}}^{L}\right)+k_{2} \mu_{\tilde{A}}^{U}\left(1-\mu_{\tilde{A}}^{U}-v_{\tilde{A}}^{U}\right)
$$

Example 12. Let $\tilde{A}_{1}=[0.08,0.16],[0.17,0.19]$ and $\tilde{A}_{2}=[0.10,0.14],[0.10,0.26]$ be two IVIFVs. By applying $\operatorname{GIS}(\tilde{A})$ presented in Equation (14), score values are calculated as $\operatorname{GIS}\left(\tilde{A}_{1}\right)=\operatorname{GIS}\left(\tilde{A}_{2}\right)=-0.04$. Therefore, it is not known which IVIFV is bigger. Moreover, if $\mu_{\tilde{A}}^{L}=\mu_{\tilde{A}}^{U}=0$, then whatever $v_{\tilde{A}}^{L}, v_{\tilde{A}}^{U}, k_{1}$ and $k_{2}$ are $\operatorname{GIS}(\tilde{A})$ is always equal to 0 .

\subsection{2. Şahin [23]'s Ranking Method of the IVIFV}

Şahin [23] proposed an accuracy function $K($.) by taking into account the hesitant degree of IVIFV

$$
K(\tilde{A})=\frac{\mu_{\tilde{A}}^{L}+\mu_{\tilde{A}}^{U}\left(1-\mu_{\tilde{A}}^{L}-v_{\tilde{A}}^{L}\right)+\mu_{\tilde{A}}^{U}+\mu_{\tilde{A}}^{L}\left(1-\mu_{\tilde{A}}^{U}-v_{\tilde{A}}^{U}\right)}{2} .
$$

Example 13. If corresponding to two different IVIFVs $\tilde{A}_{1}=[0,0],[0.27,0.37]$ and $\tilde{A}_{2}=[0,0],[0.19,0.45]$. Using Equation (15), accuracy values are obtained as $I\left(\tilde{A}_{1}\right)=I\left(\tilde{A}_{2}\right)=0$. When $\mu_{\tilde{A}}^{L}=\mu_{\tilde{A}}^{U}=0$, then $K(\tilde{A})=0$ , $v_{\tilde{A}}^{L}$ and $v_{\tilde{A}}^{U}$ have no effect on $K(\tilde{A})$.

\subsection{Nayagam et al. [10]'s Ranking Method of the IVIFV}

Nayagam et al. [10] introduced a non-hesitance score function $J($.$) to cope with the shortcomings of$ familiar methods

$$
J(\tilde{A})=\frac{\mu_{\tilde{A}}^{L}+\mu_{\tilde{A}}^{U}+v_{\tilde{A}}^{L}-v_{\tilde{A}}^{U}+\mu_{\tilde{A}}^{L} \mu_{\tilde{A}}^{U}+v_{\tilde{A}}^{L} v_{\tilde{A}}^{U}}{3} .
$$

Example 14. Let $\tilde{A}_{1}=[0.15,0.25],[0.30,0.55]$ and $\tilde{A}_{2}=[0.05,0.15],[0.50,0.55]$ be two IVIFVs, and $\tilde{A}_{1} \supset \tilde{A}_{2}$. Using Equation (16), score values are obtained as $J\left(\tilde{A}_{1}\right)=0.118$ and $I\left(\tilde{A}_{2}\right)=0.144$, and so, the Nayagam et al. [10]'s score function is unsuccessful.

\subsection{Wang and Chen [16]'s Ranking Method of the IVIFV}

The score function $S_{W C}($.$) was defined by Wang and Chen [16] as follows$ 


$$
S_{W C}(\tilde{A})=\frac{\mu_{\tilde{A}}^{L}+\mu_{\tilde{A}}^{U}+\sqrt{\mu_{\tilde{A}}^{U} v_{\tilde{A}}^{U}}\left(1-\mu_{\tilde{A}}^{L}-v_{\tilde{A}}^{L}\right)}{2}+\sqrt{\mu_{\tilde{A}}^{L} v_{\tilde{A}}^{L}}\left(1-\mu_{\tilde{A}}^{U}-v_{\tilde{A}}^{U}\right)
$$

Considering the Example 17, it is observed that in this function also if $\mu_{\tilde{A}}^{L}=\mu_{\tilde{A}}^{U}=0$ then $S_{W C}()=$.0 . This function has drawbacks for ranking.

\subsection{Zhang and Xu [24]'s Ranking Method of the IVIFV}

Zhang ve $\mathrm{Xu}[24]$ suggested an accuracy function $F($.$) .$

$$
F(\tilde{A})=\frac{1}{2}\left(\frac{\left(\mu_{\tilde{A}}^{L}-v_{\tilde{A}}^{L}\right)+\left(\mu_{\tilde{A}}^{U}-v_{\tilde{A}}^{U}\right)\left(1-\mu_{\tilde{A}}^{L}-v_{\tilde{A}}^{L}\right)}{2}+\frac{\left(\mu_{\tilde{A}}^{U}-v_{\tilde{A}}^{U}\right)+\left(\mu_{\tilde{A}}^{L}-v_{\tilde{A}}^{L}\right)\left(1-\mu_{\tilde{A}}^{U}-v_{\tilde{A}}^{U}\right)}{2}\right)
$$

Example 15. Let $\tilde{A}_{1}=[0.11,0.21],[0.11,0.21]$ and $\tilde{A}_{2}=[0.07,0.33],[0.07,0.33]$ be two IVIFVs. Using Equation (18), $F\left(\tilde{A}_{1}\right)=F\left(\tilde{A}_{2}\right)=0$ is obtained. That is, if $\mu_{\tilde{A}}^{L}=v_{\tilde{A}}^{L}$ and $\mu_{\tilde{A}}^{U}=v_{\tilde{A}}^{U}$, then $F(\tilde{A})=0$. Therefore, it cannot distinguish the IVIFVs.

\subsection{Joshi and Kumar [25]'s Ranking Method of the IVIFV}

Joshi and Kumar [25] suggest the accuracy function $T($.$) to distinguish IVIFVs.$

$$
T(\tilde{A})=\frac{\mu_{\tilde{A}}^{L}\left(1-v_{\tilde{A}}^{L}\right)+\mu_{\tilde{A}}^{U}\left(1-v_{\tilde{A}}^{U}\right)}{2}
$$

Example 16. Let $\tilde{A}_{1}=[0.45,0.67],[0.14,0.26]$ and $\tilde{A}_{2}=[0.50,0.62],[0.09,0.31]$ be two IVIFVs. By applying $T(\tilde{A})$ presented in Equation (18) we get $T\left(\tilde{A}_{1}\right)=T\left(\tilde{A}_{2}\right)=0.441$. Therefore, it is not known which IVIFV is bigger. Moreover, if $\mu_{\tilde{A}}^{L}=\mu_{\tilde{A}}^{U}=0$, then whatever $v_{\tilde{A}}^{L}, v_{\tilde{A}}^{U}, k_{1}$ and $k_{2}$ are $T(\tilde{A})$ is always equal to 0 .

\subsection{Wang and Chen [17]'s Ranking Method of the IVIFV}

Wang and Chen [23] offered a score function $S_{N W C}($.$) and an accuracy function H_{N W C}($.$) of IVIFVs.$

$$
\begin{aligned}
& S_{N W C}(\tilde{A})=\frac{\left(\mu_{\tilde{A}}^{L}+\mu_{\tilde{A}}^{U}\right)\left(\mu_{\tilde{A}}^{L}+v_{\tilde{A}}^{L}\right)}{2}-\frac{\left(v_{\tilde{A}}^{L}+v_{\tilde{A}}^{U}\right)\left(\mu_{\tilde{A}}^{U}+v_{\tilde{A}}^{U}\right)}{2} \\
& H_{N W C}(\tilde{A})=\frac{\left(1-\mu_{\tilde{A}}^{L}+\mu_{\tilde{A}}^{U}\right)\left(1-\mu_{\tilde{A}}^{L}-v_{\tilde{A}}^{L}\right)}{2}+\frac{\left(1-v_{\tilde{A}}^{L}+v_{\tilde{A}}^{U}\right)\left(1-\mu_{\tilde{A}}^{U}-v_{\tilde{A}}^{U}\right)}{2}
\end{aligned}
$$

Example 17. Let $\tilde{A}_{1}=[0.15,0.25],[0.18,0.30]$ and $\tilde{A}_{2}=[0.35,0.45],[0.38,0.50]$ be two IVIFVs and $\tilde{A}_{1} \subset \tilde{A}_{2}$. Using Equations (20) and (21), score values and accuracy values are calculated respectively as $S_{N W C}\left(\tilde{A}_{1}\right)=-0.07, S_{N W C}\left(\tilde{A}_{2}\right)=-0.13$ and $H_{N W C}\left(\tilde{A}_{1}\right)=0.62, \quad H_{N W C}\left(\tilde{A}_{2}\right)=0.18$. Both functions rank IVIFVs as $\tilde{A}_{1}>\tilde{A}_{2}$ and fail to present the expected ranking. Also, it is important mentioning that when $\mu_{\tilde{A}}^{L}=\mu_{\tilde{A}}^{U}=v_{\tilde{A}}^{L}=v_{\tilde{A}}^{U}, S_{N W C}(\tilde{A})$ and $H_{N W C}(\tilde{A})$ cannot rank IVIFVs. 


\subsection{Gong and Ma [18]'s Ranking Method of the IVIFV}

Gong and Ma [18] developed a score function $S_{G M}($.$) and an accuracy function H_{G M}($.$) of the IVIFV as$ below

$$
\begin{aligned}
& S_{G M}(\tilde{A})=\frac{v_{\tilde{A}}^{U}+v_{\tilde{A}}^{L}-\mu_{\tilde{A}}^{U}-\mu_{\tilde{A}}^{L}}{2}+\frac{\mu_{\tilde{A}}^{L}+\mu_{\tilde{\tilde{A}}}^{U}+2\left(\mu_{\tilde{A}}^{L} \mu_{\tilde{\tilde{A}}}^{U}-v_{\tilde{\tilde{A}}}^{L} v_{\tilde{A}}^{U}\right)}{\mu_{\tilde{A}}^{L}+\mu_{\tilde{A}}^{U}+v_{\tilde{A}}^{L}+v_{\tilde{A}}^{U}}, \\
& H_{G M}(\tilde{A})=\mu_{\tilde{A}}^{U}+v_{\tilde{A}}^{U}-\frac{\left(\mu_{\tilde{A}}^{U}-\mu_{\tilde{A}}^{L}\right)^{2}}{2 \mu_{\tilde{A}}^{U}}-\frac{\left(v_{\tilde{A}}^{U}-v_{\tilde{A}}^{L}\right)^{2}}{2 v_{\tilde{A}}^{U}} .
\end{aligned}
$$

Example 18. If corresponding to two different IVIFVs $\tilde{A}_{1}=[0.18,0.27],[0.19,0.40]$ and $\tilde{A}_{2}=[0.13,0.32],[0.29,0.30]$, and $\tilde{A}_{1} \subset \tilde{A}_{2}$. Using Equations (22) and (23), score values and accuracy values are obtained respectively as $S_{G M}\left(\tilde{A}_{1}\right)=0.45, S_{G M}\left(\tilde{A}_{2}\right)=0.42$ and $H_{G M}\left(\tilde{A}_{1}\right)=0.64, H_{G M}\left(\tilde{A}_{2}\right)=0.60$ . It is clear that these functions aren't valid sufficiently for the ranking of IVIFVs.

\subsection{Jia et al. [19]'s Ranking Method of the IVIFV}

Jia et al. [19] introduced a score function $J($.$) in Equation (24) utilized to quantify the information included$ in IVIFV with the idea of p-norm.

$$
J(\tilde{A})=\left[\frac{e^{\frac{\mu_{\tilde{A}}^{U}-v_{\tilde{A}}^{U}+\mu_{\tilde{A}}^{L}-v_{\tilde{A}}^{L}}{2}}-1}{e^{\frac{\mu_{\tilde{A}}^{U}-v_{\tilde{A}}^{U}+\mu_{\tilde{A}}^{L}-v_{\tilde{A}}^{L}}{2}}+1}\right]\left[\frac { 1 } { 2 ^ { \frac { 1 } { p } } + 1 } \left[\left(\left[\begin{array}{l}
\left.\left.\left.\left(\frac{\left(\mu_{\tilde{A}}^{L}\right)^{p}+\left(\mu_{\tilde{A}}^{U}\right)^{p}+\left(v_{\tilde{A}}^{L}\right)^{p}+\left(v_{\tilde{A}}^{U}\right)^{p}}{2}\right)+\left(\frac{\left(\mu_{\tilde{A}}^{L}+v_{\tilde{A}}^{L}\right)^{p}+\left(\mu_{\tilde{A}}^{U}+v_{\tilde{A}}^{U}\right)^{p}}{2}\right)\right)^{\frac{1}{p}}+\right]\right] \\
2
\end{array}\right]\right]\right.\right.
$$

Example 19. Let $\tilde{A}_{1}=[0.02,0.03],[0.02,0.03]$ and $\tilde{A}_{2}=[0.41,0.44],[0.41,0.44]$ be two IVIFVs. By applying $J(\tilde{A})$ presented in Equation (24), score values are obtained as $J\left(\tilde{A}_{1}\right)=J\left(\tilde{A}_{2}\right)=0$. Hence, when $\mu_{\tilde{A}}^{L}=v_{\tilde{A}}^{L}$ and $\mu_{\tilde{A}}^{U}=v_{\tilde{A}}^{U}$, then $J(\tilde{A})=0$ and it and cannot distinguish such IVIFVs.

\section{A NEW SCORE FUNCTION FOR IVIFSs}

Let $\tilde{A}=\left[\mu_{\tilde{A}}^{L}, \mu_{\tilde{A}}^{U}\right],\left[\left(v_{\tilde{A}}^{L}, v_{\tilde{A}}^{U}\right]\right.$ be an IVIFV. The score function $M K(\tilde{A})$ and the properties of the new score function of IVIFV are explained as below

$$
M K(\tilde{A})=\frac{\left(\mu_{\tilde{A}}^{L}+2 \mu_{\tilde{A}}^{U}-v_{\tilde{A}}^{U}-1\right)+\left(v_{\tilde{A}}^{U}\right)\left(1-\mu_{\tilde{A}}^{U}-v_{\tilde{A}}^{L}\right)}{2} .
$$

Property 1. $M K(\tilde{A})=1$ if and only if $\tilde{A}=([1,1],[0,0])$.

Proof 1. If $M K(\tilde{A})=1$, then it is obtained that $\mu_{\tilde{A}}^{L}+2 \mu_{\tilde{A}}^{U}-\mu_{\tilde{A}}^{U} v_{\tilde{A}}^{U}-v_{\tilde{A}}^{L} v_{\tilde{A}}^{U}=3$. In order to provide this equality under condition $0 \leq \mu_{\tilde{A}}^{L}, \mu_{\tilde{A}}^{U}, v_{\tilde{A}}^{L}, v_{\tilde{A}}^{U} \leq 1$, then $\mu_{\tilde{A}}^{L}=1,2 \mu_{\tilde{A}}^{U}=2, \mu_{\tilde{A}}^{U} v_{\tilde{A}}^{U}=0$ and $v_{\tilde{A}}^{L} v_{\tilde{A}}^{U}=0$. It can be easily observed that $\tilde{A}=([1,1],[0,0])$ provides these equations.

Property 2. $M K(\tilde{A})=-1$ if and only if $\tilde{A}=([0,0],[1,1])$. 
Proof 2. If $M K(\tilde{A})=-1$, then it is obtained that $\mu_{\tilde{A}}^{L}+2 \mu_{\tilde{A}}^{U}-\mu_{\tilde{A}}^{U} v_{\tilde{A}}^{U}-v_{\tilde{A}}^{L} v_{\tilde{A}}^{U}=-1$. In order to provide this equality under condition $0 \leq \mu_{\tilde{A}}^{L}, \mu_{\tilde{A}}^{U}, v_{\tilde{A}}^{L}, v_{\tilde{A}}^{U} \leq 1$, then $\mu_{\tilde{A}}^{L}=0,2 \mu_{\tilde{A}}^{U}=0, \mu_{\tilde{A}}^{U} v_{\tilde{A}}^{U}=0$ and $v_{\tilde{A}}^{L} v_{\tilde{A}}^{U}=1$. It can be easily observed that $\tilde{A}=([0,0],[1,1])$ provides these equations.

Property 3. $M K(\tilde{A})$ has monotonicity property, i.e. $M K(\tilde{A})$ on $\mu_{\tilde{A}}^{L}$ and $\mu_{\tilde{A}}^{U}$ monotonously increases; $M K(\tilde{A})$ on $v_{\tilde{A}}^{L}$ and $v_{\tilde{A}}^{U}$ monotonously decreases.

Proof 3. When the partial derivatives of the $M K(\tilde{A})$ with respect to $\mu_{\tilde{A}}^{L}, \mu_{\tilde{A}}^{U}, v_{\tilde{A}}^{L}$ and $v_{\tilde{A}}^{U}$ are examined, it is seen that $M K(\tilde{A})$ provides monotonicity property

$$
\frac{\partial M K(\tilde{A})}{\partial \mu_{\tilde{A}}^{L}}=\frac{1}{2}>0, \frac{\partial M K(\tilde{A})}{\partial \mu_{\tilde{A}}^{U}}=\frac{2-v_{\tilde{A}}^{U}}{2}>0, \frac{\partial M K(\tilde{A})}{\partial v_{\tilde{A}}^{L}}=\frac{-v_{\tilde{A}}^{U}}{2}<0, \frac{\partial M K(\tilde{A})}{\partial v_{\tilde{A}}^{U}}=\frac{-v_{\tilde{A}}^{L}}{2}<0 .
$$

Therefore, if $\mu_{\tilde{A}}^{L}>\mu_{\tilde{B}}^{L}, \mu_{\tilde{A}}^{U}>\mu_{\tilde{B}}^{U}, v_{\tilde{A}}^{L}<v_{\tilde{B}}^{L}$ and $v_{\tilde{A}}^{U}<v_{\tilde{B}}^{U}$ then $M K(\tilde{A})>M K(\tilde{B})$.

Property 4. Let $\tilde{A}$ and $\tilde{B}$ be two IVIFVs. $M K(\tilde{A})=M K(\tilde{B})$ if and only if $\tilde{A}=\tilde{B}$.

Proof 4. If $M K(\tilde{A})=M K(\tilde{B})$, then we obtain

$$
\left(\mu_{\tilde{A}}^{L}+2 \mu_{\tilde{A}}^{U}-\mu_{\tilde{A}}^{U} v_{\tilde{A}}^{U}-v_{\tilde{A}}^{L} v_{\tilde{A}}^{U}-1\right) / 2=\left(\mu_{\tilde{B}}^{L}+2 \mu_{\tilde{B}}^{U}-\mu_{\tilde{B}}^{U} v_{\tilde{B}}^{U}-v_{\tilde{B}}^{L} v_{\tilde{B}}^{U}-1\right) / 2 \text {. }
$$

It is obtained that $\mu_{\tilde{A}}^{L}=\mu_{\tilde{B}}^{L}, \mu_{\tilde{A}}^{U}=\mu_{\tilde{B}}^{U}, \mu_{\tilde{A}}^{U} v_{\tilde{A}}^{U}=\mu_{\tilde{B}}^{U} v_{\tilde{B}}^{U}, v_{\tilde{A}}^{L} v_{\tilde{A}}^{U}=v_{\tilde{B}}^{L} v_{\tilde{B}}^{U}$ from above equality. Since $\mu_{\tilde{A}}^{U}=\mu_{\tilde{B}}^{U}$, then $v_{\tilde{A}}^{U}=v_{\tilde{B}}^{U}$ from $\mu_{\tilde{A}}^{U} v_{\tilde{A}}^{U}=\mu_{\tilde{B}}^{U} v_{\tilde{B}}^{U}$. Moreover, since $v_{\tilde{A}}^{U}=v_{\tilde{B}}^{U}$ then $v_{\tilde{A}}^{L}=v_{\tilde{B}}^{L}$ from $v_{\tilde{A}}^{L} v_{\tilde{A}}^{U}=v_{\tilde{B}}^{L} v_{\tilde{B}}^{U}$. Evidently, if $\mu_{\tilde{A}}^{L}=\mu_{\tilde{B}}^{L}, \mu_{\tilde{A}}^{U}=\mu_{\tilde{B}}^{U}, v_{\tilde{A}}^{L}=v_{\tilde{B}}^{L}$ and $v_{\tilde{A}}^{U}=v_{\tilde{B}}^{U}$ then $\tilde{A}=\tilde{B}$, and so, if $\tilde{A}=\tilde{B}$ then $M K(\tilde{A})=M K(\tilde{B})$.

\section{A COMPARISON OF RANKING FUNCTIONS FOR IVIFS}

Properties of the ranking functions mentioned in Section 3 such as monotonicity, max value, and min value are summarized in Tables 1 and 2. While the value range of the score function and accuracy function developed by $\mathrm{Xu}$ is $[-1,1]$ and [0.1], respectively, it is observed that the value ranges of the score functions and accuracy functions developed later differed. While the maximum values obtained by accuracy ranking functions in [17, 24] and score function [19] are smaller than 1, the maximum value obtained by score function [13] is bigger than 1 . Besides, minimum and maximum values calculated by accuracy functions in $[12,15,17,18]$ are equal, and this equality is unacceptable.

According to the containment relation of IVIFVs, the score function should monotonically increase, while the accuracy function should monotonically decrease [18]. The monotonicity properties of the ranking functions are examined with partial derivatives by considering Definition 5 and Definition 6 . The partial derivatives results illustrated that the ranking functions in $[6,7,9,10,13,17,20-25]$ do not satisfy the monotonicity property. On the other hand, the proposed score function satisfies the monotonicity property.

In order to compare ranking functions and show the superiority of the new score function, the comparative analysis is conducted by using twenty IVIFV pairs compiled from several studies $[4,5,7,10,18,20,23$, 25-29]. Using the new score function and the existing ranking functions, ranking values are calculated. These values are shown in respectively Tables 3 and 4 . It is seen from Tables 3 and 4, that the existing ranking functions produce counter-intuitive results for two different IVIFVs. When all examples are investigated, the proposed score function is the only one that does not yield counter-intuitive cases. Hence, 
it is determined that the new score function can distinguish the IVIF values that the existing ranking functions are not capable of distinguishing.

Table 1. Properties of the score functions

\begin{tabular}{lllcc}
\multicolumn{2}{l}{ Score Function } & Monotonicity & Minimum Value & Maximum Value \\
\hline S(.) & {$[12]$} & Monotonic & -1 & 1 \\
S. $()$. & {$[13]$} & Nonmonotonic & 0 & 4 \\
Do(.) & {$[6]$} & Nonmonotonic & 0 & 1 \\
Dp(.) & {$[6]$} & Nonmonotonic & 0 & 1 \\
I(.) & {$[14]$} & Monotonic & 0 & 1 \\
W $_{\mathrm{S}}()$. & {$[15]$} & Monotonic & -1 & 1 \\
GIS(.) & {$[9]$} & Nonmonotonic & 0 & 1 \\
$\mathrm{~J}()$. & {$[10]$} & Nonmonotonic & 0.33 & 1 \\
$\mathrm{~S}_{\mathrm{WC}}()$. & {$[16]$} & Monotonic & 0 & 1 \\
$\mathrm{~S}_{\mathrm{NWC}}()$. & {$[17]$} & Nonmonotonic & -1 & 1 \\
$\mathrm{~S}_{\mathrm{GM}}()$. & {$[18]$} & Monotonic & 0 & 1 \\
$\mathrm{~J}()$. & {$[19]$} & Monotonic & -0.46 & 0.46 \\
\hline
\end{tabular}

Table 2. Properties of the accuracy functions

\begin{tabular}{|c|c|c|c|c|}
\hline \multicolumn{2}{|c|}{ Accuracy Function } & \multirow{2}{*}{$\begin{array}{l}\text { Monotonicity } \\
\text { Monotonic }\end{array}$} & \multirow{2}{*}{$\frac{\text { Minimum Value }}{\mathbf{1}}$} & \multirow{2}{*}{$\frac{\text { Maximum Value }}{\mathbf{1}}$} \\
\hline $\mathrm{H}()$. & {$[12]$} & & & \\
\hline $\mathrm{M}()$. & [5] & Monotonic & 0 & 1 \\
\hline $\mathrm{L}()$. & [20] & Nonmonotonic & -1 & 1 \\
\hline $\mathrm{LG}()$. & [21] & Nonmonotonic & 0 & 1 \\
\hline $\mathrm{W}_{\mathrm{H}}()$. & [15] & Monotonic & 1 & 1 \\
\hline $\mathrm{P}()$. & [7] & Nonmonotonic & -0.33 & 1 \\
\hline $\mathrm{H}_{\mathrm{K}}()$. & [22] & Nonmonotonic & -1 & 1 \\
\hline $\mathrm{K}()$. & [23] & Nonmonotonic & 0 & 1 \\
\hline $\mathrm{F}()$. & [24] & Nonmonotonic & -0.50 & 0.50 \\
\hline $\mathrm{T}()$. & [25] & Nonmonotonic & 0 & 1 \\
\hline $\mathrm{H}_{\mathrm{NWC}}()$. & {$[17]$} & Nonmonotonic & 0 & 0 \\
\hline $\mathrm{H}_{\mathrm{GM}}()$. & [18] & Monotonic & 1 & 1 \\
\hline
\end{tabular}

In order to compare ranking functions and show the superiority of the new score function, the comparative analysis is conducted by using twenty IVIFV pairs compiled from several studies $[4,5,7,10,18,20,23$, 25-29]. Using the new score function and the existing ranking functions, ranking values are calculated. These values are shown in respectively Tables 3 and 4 . It is seen from Tables 3 and 4, that the existing ranking functions produce counter-intuitive results for two different IVIFVs. When all examples are investigated, the proposed score function is the only one that does not yield counter-intuitive cases. Hence, it is determined that the new score function can distinguish the IVIF values that the existing ranking functions are not capable of distinguishing.

The theorems are presented below to show that the cases described with examples in Tables 3 and 4 are not limited to the examples selected in this study. In these theorems, it is proved that the proposed score function overcomes the limited situations that the existing ranking functions have.

Theorem 1. Ranking function $D_{o}$ (.) proposed by Tu and Chen [6] produces equal score value when $\mu_{\tilde{A}}^{L}=\mu_{\tilde{B}}^{L}$ and $v_{\tilde{A}}^{U}=v_{\tilde{B}}^{U}$ without affecting $\mu_{\tilde{A}}^{U}, \mu_{\tilde{B}}^{U}, v_{\tilde{A}}^{L}$ and $v_{\tilde{B}}^{L}$. But, $M K(\tilde{A}) \neq M K(\tilde{B})$ when $\mu_{\tilde{A}}^{L}=\mu_{\tilde{B}}^{L}$ and $v_{\tilde{A}}^{U}=v_{\tilde{B}}^{U}$ under the condition $\mu_{\tilde{A}}^{U} \neq \mu_{\tilde{B}}^{U}$ and $v_{\tilde{A}}^{L} \neq v_{\tilde{B}}^{L}$.

Proof. In case $\mu_{\tilde{A}}^{L}=\mu_{\tilde{B}}^{L}$ and $v_{\tilde{A}}^{U}=v_{\tilde{B}}^{U}$, then we obtain $M K(\tilde{A})=\left(\mu_{\tilde{A}}^{L}+2 \mu_{\tilde{A}}^{U}-\mu_{\tilde{A}}^{U} v_{\tilde{A}}^{U}-v_{\tilde{A}}^{L} v_{\tilde{A}}^{U}-1\right) / 2$ and $M K(\tilde{B})=\left(\mu_{\tilde{A}}^{L}+2 \mu_{\tilde{B}}^{U}-\mu_{\tilde{B}}^{U} v_{\tilde{A}}^{U}-v_{\tilde{B}}^{L} v_{\tilde{A}}^{U}-1\right) / 2$. If $M K(\tilde{A}) \neq M K(\tilde{B})$ then $\left(\mu_{\tilde{A}}^{L}+2 \mu_{\tilde{A}}^{U}-\mu_{\tilde{A}}^{U} v_{\tilde{A}}^{U}-v_{\tilde{A}}^{L} v_{\tilde{A}}^{U}-1\right) / 2 \neq\left(\mu_{\tilde{A}}^{L}+2 \mu_{\tilde{B}}^{U}-\mu_{\tilde{B}}^{U} v_{\tilde{A}}^{U}-v_{\tilde{B}}^{L} v_{\tilde{A}}^{U}-1\right) / 2$. When this inequality is simplified as $\left(2 \mu_{\tilde{A}}^{U}-v_{\tilde{A}}^{U}\left(\mu_{\tilde{A}}^{U}+v_{\tilde{A}}^{L}\right)\right) \neq\left(2 \mu_{\tilde{B}}^{U}-v_{\tilde{A}}^{U}\left(\mu_{\tilde{B}}^{U}+v_{\tilde{B}}^{L}\right)\right)$ it is seen that $M K(\tilde{A}) \neq M K(\tilde{B})$ if $\mu_{\tilde{A}}^{U} \neq \mu_{\tilde{B}}^{U}$ and $v_{\tilde{A}}^{L} \neq v_{\tilde{B}}^{L}$. 
Theorem 2. Ranking function $D_{p}(\tilde{A})$ proposed by Tu and Chen [6] produces equal score value when $\mu_{\tilde{A}}^{U}=\mu_{\tilde{B}}^{U}$ and $v_{\tilde{A}}^{L}=v_{\tilde{B}}^{L}$ without affecting $\mu_{\tilde{A}}^{L}, \mu_{\tilde{B}}^{L}, v_{\tilde{A}}^{U}$ and $v_{\tilde{B}}^{U}$. But $M K(\tilde{A}) \neq M K(\tilde{B})$ when $\mu_{\tilde{A}}^{U}=\mu_{\tilde{B}}^{U}$ and $v_{\tilde{A}}^{L}=v_{\tilde{B}}^{L}$ under the condition $\mu_{\tilde{A}}^{L} \neq \mu_{\tilde{B}}^{L}$ and $v_{\tilde{A}}^{U} \neq v_{\tilde{B}}^{U}$.

Proof. In case $\mu_{\tilde{A}}^{U}=\mu_{\tilde{B}}^{U}$ and $v_{\tilde{A}}^{L}=v_{\tilde{B}}^{L}$, then we obtain $M K(\tilde{A})=\left(\mu_{\tilde{A}}^{L}+2 \mu_{\tilde{A}}^{U}-\mu_{\tilde{A}}^{U} v_{\tilde{A}}^{U}-v_{\tilde{A}}^{L} v_{\tilde{A}}^{U}-1\right) / 2$ and $M K(\tilde{B})=\left(\mu_{\tilde{B}}^{L}+2 \mu_{\tilde{A}}^{U}-\mu_{\tilde{A}}^{U} v_{\tilde{B}}^{U}-v_{\tilde{A}}^{L} v_{\tilde{B}}^{U}-1\right) / 2$. If $M K(\tilde{A}) \neq M K(\tilde{B})$ then $\left(\mu_{\tilde{A}}^{L}+2 \mu_{\tilde{A}}^{U}-\mu_{\tilde{A}}^{U} v_{\tilde{A}}^{U}-v_{\tilde{A}}^{L} v_{\tilde{A}}^{U}-1\right) / 2 \neq\left(\mu_{\tilde{B}}^{L}+2 \mu_{\tilde{A}}^{U}-\mu_{\tilde{A}}^{U} v_{\tilde{B}}^{U}-v_{\tilde{A}}^{L} v_{\tilde{B}}^{U}-1\right) / 2$. When this inequality is simplified as $\left(\mu_{\tilde{A}}^{L}-v_{\tilde{A}}^{U}\left(\mu_{\tilde{A}}^{U}+v_{\tilde{A}}^{L}\right)\right) \neq\left(\mu_{\tilde{B}}^{L}-v_{\tilde{B}}^{U}\left(\mu_{\tilde{A}}^{U}+v_{\tilde{A}}^{L}\right)\right)$ it is seen that $M K(\tilde{A}) \neq M K(\tilde{B})$ if $\mu_{\tilde{A}}^{L} \neq \mu_{\tilde{B}}^{L}$ and $v_{\tilde{A}}^{U} \neq v_{\tilde{B}}^{U}$.

Theorem 3. The score functions in $[12,15,19]$ and accuracy function in [24] give ranking value 0 when the midpoints of the membership and the non-membership coincide, namely $\mu_{\tilde{A}}^{L}=v_{\tilde{A}}^{L}$ and $\mu_{\tilde{A}}^{U}=v_{\tilde{A}}^{U}$. But, $\operatorname{MK}(\tilde{A}) \neq 0$ when $\mu_{\tilde{A}}^{L}=v_{\tilde{A}}^{L}$ and $\mu_{\tilde{A}}^{U}=v_{\tilde{A}}^{U}$ under the condition $\mu_{\tilde{A}}^{L} \neq \mu_{\tilde{A}}^{U} \neq 0$.

Proof. When $\mu_{\tilde{A}}^{L}=v_{\tilde{A}}^{L}$ and $\mu_{\tilde{A}}^{U}=v_{\tilde{A}}^{U}$, we obtain $M K(\tilde{A})=\left(\mu_{\tilde{A}}^{L}+2 \mu_{\tilde{A}}^{U}-\left(\mu_{\tilde{A}}^{U}\right)^{2}-\mu_{\tilde{A}}^{L} \mu_{\tilde{A}}^{U}-1\right) / 2$. So, $M K(\tilde{A}) \neq 0$ when $\mu_{\tilde{A}}^{L} \neq \mu_{\tilde{A}}^{U} \neq 0$.

Theorem 4. Score functions proposed in $[6,9,14,16]$ and accuracy functions proposed in [23, 25] give ranking value 0 when $\mu_{\tilde{A}}^{L}=\mu_{\tilde{A}}^{U}=0$. So, $v_{\tilde{A}}^{L}$ or $v_{\tilde{A}}^{U}$ have no effect on ranking value. But, score value $M K(\tilde{A})$ is affected from $v_{\tilde{A}}^{L}$ and $v_{\tilde{A}}^{U}$ when $\mu_{\tilde{A}}^{L}=\mu_{\tilde{A}}^{U}=0$.

Proof. If $\mu_{\tilde{A}}^{L}=\mu_{\tilde{A}}^{U}=0$, then $M K(\tilde{A})=\left(-v_{\tilde{A}}^{L} v_{\tilde{A}}^{U}-1\right) / 2$. So, $M K(\tilde{A})$ is affected from $v_{\tilde{A}}^{L}$ and $v_{\tilde{A}}^{U}$.

\section{THE MCDM METHOD USING THE PROPOSED SCORE FUNCTION}

In this section, a MCDM algorithm is adapted from Sahin [23]'s study in order to provide the effectiveness of the new score function in MCDM problems. Two illustrative examples introduced in $[4,25]$ are used to demonstrate the application of the algorithm.

Algorithm 1. Step 1: Compute the aggregated IVIFVs $\gamma_{i}$ for each alternative $A_{i} \quad(i=1,2, \ldots, m)$ using IVIFWA operator considering criteria' weights $w_{j}(j=1,2, \ldots, n)$ according to each row in decision matrix $R_{m \times n}$.

Step 2: Calculate the ranking value for the aggregated IVIF value $\gamma_{i}(i=1,2, \ldots, m)$ using ranking function. Step 3: Rank the alternatives $A_{1}, A_{2}, \ldots, A_{m}$ by using their ranking values and select the best alternative according to higher values.

Example 20. [4] There are four possible alternatives for company investment: $\left(A_{1}\right)$ car company, $\left(A_{2}\right)$ food company, $\left(\mathrm{A}_{3}\right)$ computer company, and $\left(\mathrm{A}_{4}\right)$ arms company. The company wants to make a decision considering five criteria: (C1) productivity, (C2) technological innovation capability, (C3) marketing capability, (C4) management, and (C5) risk avoidance. The criteria' weights are $w=[0.2,0.3,0.15,0.1,0.25]^{T}$. The decision matrix $R_{4 \times 5}$ (Table 5) includes information related to the evaluation of alternatives on criteria. 
Table 3.The comparison of the score functions (counter-intuitive cases are in bold type)

\begin{tabular}{|c|c|c|c|c|c|c|c|c|c|c|c|c|c|c|c|}
\hline \multicolumn{2}{|c|}{ IVIF Values } & \multirow{2}{*}{$\begin{array}{c}\text { Rank } \\
1\end{array}$} & \multirow{2}{*}{$\begin{array}{c}\mathrm{Xu} \\
{[12]} \\
-0.200\end{array}$} & \multirow{2}{*}{$\begin{array}{c}\begin{array}{c}\text { Lee } \\
{[13]}\end{array} \\
1.000\end{array}$} & \multirow{2}{*}{$\begin{array}{c}\text { Tu and } \\
\text { Chen,1 [6] } \\
0.120\end{array}$} & \multirow{2}{*}{$\begin{array}{c}\text { Tu and } \\
\text { Chen,2 [6] }\end{array}$} & \multirow{2}{*}{$\begin{array}{c}\text { Bai } \\
{[14]}\end{array}$} & \multirow{2}{*}{$\begin{array}{l}\text { Wang and } \\
\text { Niu [15] } \\
-0.260\end{array}$} & \multirow{2}{*}{$\begin{array}{c}\text { Garg } \\
{[9]}\end{array}$} & \multirow{2}{*}{$\begin{array}{l}\text { Nayagam } \\
\text { et al. [10] } \\
\mathbf{0 . 1 4 7}\end{array}$} & \multirow{2}{*}{$\begin{array}{c}\text { Wang and } \\
\text { Chen [16] } \\
0.368\end{array}$} & \multirow{2}{*}{$\begin{array}{c}\text { Wang and } \\
\text { Chen [17] } \\
-0.280\end{array}$} & \multirow{2}{*}{$\begin{array}{c}\text { Gong and } \\
\text { Ma [18] }\end{array}$} & \multirow{2}{*}{$\begin{array}{c}\text { Jia et al. } \\
{[19]} \\
-0.054\end{array}$} & \multirow{2}{*}{$\begin{array}{c}\text { This } \\
\text { study } \\
-0.280\end{array}$} \\
\hline Al & {$[0.20,0.30],[0.30,0.60]$} & & & & & & & & & & & & & & \\
\hline$B 1$ & {$[0.10,0.20],[0.50,0.60]$} & 2 & -0.400 & 0.750 & 0.070 & 0.450 & 0.190 & -0.520 & 0.190 & 0.173 & 0.242 & -0.350 & 0.214 & -0.118 & -0.460 \\
\hline$A 2$ & {$[0.30,0.60],[0.20,0.30]$} & 1 & 0.200 & 1.500 & 0.330 & 0.880 & 0.555 & 0.260 & 0.555 & 0.347 & 0.568 & 0.000 & 0.614 & 0.054 & 0.130 \\
\hline$B 2$ & {$[0.40,0.50],[0.10,0.40]$} & 2 & 0.200 & 1.500 & 0.320 & 0.910 & 0.575 & 0.260 & 0.575 & 0.280 & 0.572 & 0.000 & 0.671 & 0.052 & 0.080 \\
\hline$A 3$ & {$[0.40,0.50],[0.20,0.30]$} & 2 & 0.200 & 1.500 & 0.400 & 0.840 & 0.580 & 0.260 & 0.580 & 0.353 & 0.556 & 0.070 & 0.643 & 0.052 & 0.095 \\
\hline B3 & {$[0.50,0.60],[0.10,0.20]$} & 1 & 0.400 & 1.750 & 0.550 & 0.930 & 0.710 & 0.520 & 0.710 & 0.440 & 0.642 & 0.210 & 0.786 & 0.118 & 0.280 \\
\hline A4 & {$[0.18,0.60],[0.10,0.30]$} & 1 & 0.190 & 1.308 & 0.220 & 0.930 & 0.485 & 0.268 & 0.485 & 0.239 & 0.549 & -0.071 & 0.603 & 0.048 & 0.085 \\
\hline$B 4$ & {$[0.28,0.50],[0.00,0.40]$} & 2 & 0.190 & 1.308 & 0.258 & 1.000 & 0.516 & 0.268 & 0.516 & 0.173 & 0.551 & -0.071 & 0.708 & 0.044 & 0.040 \\
\hline A5 & {$[0.50,0.60],[0.10,0.30]$} & 2 & 0.350 & 1.800 & 0.450 & 0.930 & 0.680 & 0.438 & 0.680 & 0.410 & 0.646 & 0.150 & 0.743 & 0.106 & 0.245 \\
\hline B5 & {$[0.60,0.70],[0.05,0.15]$} & 1 & 0.550 & 2.067 & 0.660 & 0.973 & 0.808 & 0.688 & 0.808 & 0.543 & 0.720 & 0.338 & 0.867 & 0.183 & 0.444 \\
\hline A6 & {$[0.35,0.45],[0.20,0.30]$} & 2 & 0.150 & 1.353 & 0.368 & 0.820 & 0.535 & 0.203 & 0.535 & 0.306 & 0.516 & 0.033 & 0.615 & 0.035 & 0.028 \\
\hline B6 & {$[0.30,0.50],[0.15,0.35]$} & 1 & 0.150 & 1.353 & 0.300 & 0.873 & 0.520 & 0.203 & 0.520 & 0.268 & 0.531 & $-\mathbf{0 . 0 3 3}$ & 0.615 & 0.036 & 0.036 \\
\hline A7 & {$[0.00,0.00],[0.10,0.10]$} & 1 & -0.100 & 0.643 & 0.000 & 0.810 & 0.000 & -0.190 & 0.000 & 0.003 & 0.000 & -0.010 & 0.000 & -0.005 & -0.505 \\
\hline$B 7$ & {$[0.00,0.00],[0.90,0.90]$} & 2 & -0.900 & 0.167 & 0.000 & 0.010 & 0.000 & -0.990 & 0.000 & 0.270 & 0.000 & -0.810 & 0.000 & -0.380 & -0.905 \\
\hline A8 & {$[0.30,0.50],[0.30,0.50]$} & 2 & 0.000 & 1.429 & 0.210 & 0.790 & 0.460 & 0.000 & 0.460 & 0.300 & 0.500 & -0.160 & 0.500 & 0.000 & -0.050 \\
\hline$B 8$ & {$[0.40,0.50],[0.40,0.50]$} & 1 & 0.000 & 1.667 & 0.240 & 0.760 & 0.490 & 0.000 & 0.490 & 0.400 & 0.500 & -0.090 & 0.500 & 0.000 & -0.025 \\
\hline A9 & {$[0.40,0.60],[0.00,0.30]$} & 2 & 0.350 & 1.588 & 0.400 & 1.000 & 0.650 & 0.473 & 0.650 & 0.313 & 0.627 & 0.065 & 0.788 & 0.097 & 0.210 \\
\hline B9 & {$[0.30,0.70],[0.10,0.20]$} & 1 & 0.350 & 1.588 & 0.390 & 0.950 & 0.625 & 0.473 & 0.625 & 0.377 & 0.621 & 0.065 & 0.712 & 0.101 & 0.270 \\
\hline A10 & {$[0.00,0.00],[0.10,0.30]$} & 2 & -0.200 & 0.615 & 0.000 & 0.810 & 0.000 & -0.360 & 0.000 & -0.057 & 0.000 & -0.060 & 0.050 & -0.022 & -0.515 \\
\hline$B 10$ & {$[0.00,0.00],[0.00,0.20]$} & 1 & -0.100 & 0.643 & 0.000 & 1.000 & 0.000 & -0.190 & 0.000 & -0.067 & 0.000 & -0.020 & 0.100 & -0.007 & -0.500 \\
\hline A11 & {$[0.20,0.40],[0.20,0.40]$} & 2 & 0.000 & 1.111 & 0.200 & 0.800 & 0.400 & 0.000 & 0.400 & 0.187 & 0.440 & -0.120 & 0.500 & 0.000 & -0.120 \\
\hline$B 11$ & {$[0.15,0.45],[0.15,0.45]$} & 1 & 0.000 & 1.111 & 0.143 & 0.858 & 0.375 & 0.000 & 0.375 & 0.145 & 0.465 & -0.180 & 0.500 & 0.000 & -0.110 \\
\hline A12 & {$[0.45,0.55],[0.02,0.18]$} & 2 & 0.400 & 1.556 & 0.536 & 0.982 & 0.694 & 0.560 & 0.694 & 0.364 & 0.596 & 0.162 & 0.840 & 0.105 & 0.224 \\
\hline$B 12$ & {$[0.40,0.60],[0.01,0.19]$} & 1 & 0.400 & 1.556 & 0.488 & 0.992 & 0.681 & 0.560 & 0.681 & 0.354 & 0.606 & 0.126 & 0.830 & 0.107 & 0.242 \\
\hline A13 & {$[0.10,0.20],[0.60,0.80]$} & 2 & -0.550 & 0.692 & 0.030 & 0.400 & 0.165 & -0.633 & 0.165 & 0.200 & 0.210 & -0.595 & 0.185 & -0.202 & -0.570 \\
\hline$B 13$ & {$[0.40,0.50],[0.00,0.10]$} & 1 & 0.400 & 1.400 & 0.560 & 1.000 & 0.670 & 0.600 & 0.670 & 0.333 & 0.517 & 0.150 & 0.900 & 0.093 & 0.175 \\
\hline A14 & {$[0.10,0.20],[0.30,0.40]$} & 2 & -0.200 & 0.800 & 0.110 & 0.610 & 0.220 & -0.300 & 0.220 & 0.113 & 0.269 & -0.150 & 0.300 & -0.039 & -0.350 \\
\hline B14 & {$[0.00,0.30],[0.20,0.50]$} & 1 & -0.200 & 0.800 & 0.000 & 0.760 & 0.180 & -0.300 & 0.180 & 0.033 & 0.305 & -0.250 & 0.300 & -0.043 & -0.325 \\
\hline A15 & {$[0.13,0.26],[0.17,0.43]$} & 1 & -0.105 & 0.891 & 0.131 & 0.777 & 0.281 & -0.158 & 0.281 & 0.079 & 0.335 & -0.149 & 0.420 & -0.020 & -0.267 \\
\hline B15 & {$[0.17,0.22],[0.09,0.51]$} & 2 & -0.105 & 0.891 & 0.138 & 0.868 & 0.288 & -0.158 & 0.288 & 0.018 & 0.336 & -0.168 & 0.482 & -0.022 & -0.274 \\
\hline A16 & {$[0.20,0.20],[0.40,0.80]$} & 2 & -0.400 & 0.857 & 0.040 & 0.520 & 0.240 & -0.480 & 0.240 & 0.120 & 0.280 & -0.480 & 0.300 & -0.136 & -0.440 \\
\hline B16 & {$[0.20,0.20],[0.20,0.35]$} & 1 & -0.075 & 0.902 & 0.220 & 0.720 & 0.305 & -0.114 & 0.305 & 0.120 & 0.324 & -0.071 & 0.433 & -0.012 & -0.270 \\
\hline A17 & {$[0.20,0.30],[0.50,0.60]$} & 2 & -0.300 & 1.000 & 0.120 & 0.550 & 0.295 & -0.360 & 0.295 & 0.253 & 0.329 & -0.320 & 0.313 & -0.092 & -0.340 \\
\hline$B 17$ & {$[0.30,0.40],[0.20,0.40]$} & 1 & 0.050 & 1.235 & 0.270 & 0.800 & 0.465 & 0.067 & 0.465 & 0.233 & 0.474 & -0.065 & 0.550 & 0.010 & -0.070 \\
\hline A18 & {$[0.20,0.40],[0.20,0.30]$} & 2 & 0.050 & 1.105 & 0.240 & 0.800 & 0.420 & 0.073 & 0.420 & 0.213 & 0.434 & -0.055 & 0.532 & 0.009 & -0.090 \\
\hline$B 18$ & {$[0.30,0.50],[0.10,0.20]$} & 1 & 0.250 & 1.316 & 0.390 & 0.910 & 0.565 & 0.363 & 0.565 & 0.290 & 0.521 & 0.055 & 0.714 & 0.057 & 0.090 \\
\hline A19 & {$[0.60,0.70],[0.20,0.30]$} & 2 & 0.400 & 2.333 & 0.480 & 0.920 & 0.710 & 0.440 & 0.710 & 0.560 & 0.696 & 0.270 & 0.722 & 0.143 & 0.365 \\
\hline$B 19$ & {$[0.50,0.70],[0.10,0.20]$} & 1 & 0.450 & 1.933 & 0.550 & 0.950 & 0.735 & 0.563 & 0.735 & 0.490 & 0.686 & 0.225 & 0.790 & 0.145 & 0.370 \\
\hline A2O & {$[0.50,0.50],[0.063,0.25]$} & 1 & 0.344 & 1.593 & 0.500 & 0.941 & 0.672 & 0.461 & 0.672 & 0.360 & 0.599 & 0.164 & 0.775 & 0.093 & 0.180 \\
\hline$B 20$ & {$[0.50,0.50],[0.031,0.28]$} & 2 & 0.344 & 1.593 & 0.468 & 0.970 & 0.672 & 0.461 & 0.672 & 0.336 & 0.602 & 0.143 & 0.786 & 0.093 & 0.175 \\
\hline
\end{tabular}


Table 4. The comparison of the accuracy functions (counter-intuitive cases are in bold type)

\begin{tabular}{|c|c|c|c|c|c|c|c|c|c|c|c|c|c|c|}
\hline IVIF & Values & Rank & $\mathrm{Xu}[12]$ & $\mathrm{Ye}[5]$ & $\begin{array}{c}\text { Nayagam and } \\
\text { Sivaraman [21] }\end{array}$ & $\begin{array}{c}\text { Nayagam et al. } \\
{[20]}\end{array}$ & $\begin{array}{c}\text { Wang and } \\
\text { Niu [15] }\end{array}$ & $\begin{array}{c}\text { Kang et } \\
\text { al.[22] }\end{array}$ & $\begin{array}{c}\text { Joshi and } \\
\text { Kharayat [7] }\end{array}$ & $\begin{array}{c}\text { Şahin } \\
{[23]}\end{array}$ & $\begin{array}{c}\text { Zhang and } \\
\mathrm{Xu}[24]\end{array}$ & $\begin{array}{c}\text { Joshi and } \\
\text { Kumar [25] }\end{array}$ & $\begin{array}{l}\text { Wang and } \\
\text { Chen [17] }\end{array}$ & $\begin{array}{c}\text { Gong and } \\
\text { Ma [18] }\end{array}$ \\
\hline$A 1$ & {$[0.20,0.30],[0.30,0.60]$} & 1 & 0.700 & -0.050 & 0.400 & -0.080 & 0.910 & -0.365 & 0.127 & 0.335 & -0.118 & 0.130 & 0.340 & 0.850 \\
\hline$B 1$ & {$[0.10,0.20],[0.50,0.60]$} & 2 & 0.700 & -0.150 & 0.300 & -0.315 & 0.910 & -0.570 & 0.007 & 0.200 & -0.250 & 0.065 & 0.330 & 0.790 \\
\hline$A 2$ & {$[0.30,0.60],[0.20,0.30]$} & 1 & 0.700 & 0.150 & 0.600 & 0.320 & 0.910 & 0.115 & 0.340 & 0.615 & 0.133 & 0.330 & 0.380 & 0.850 \\
\hline$B 2$ & {$[0.40,0.50],[0.10,0.40]$} & 2 & 0.700 & 0.150 & 0.600 & 0.320 & 0.910 & 0.095 & 0.353 & 0.595 & 0.113 & 0.330 & 0.340 & 0.850 \\
\hline$A 3$ & {$[0.40,0.50],[0.20,0.30]$} & 2 & 0.700 & 0.150 & 0.600 & 0.315 & 0.910 & 0.120 & 0.347 & 0.590 & 0.125 & 0.335 & 0.330 & 0.790 \\
\hline B3 & {$[0.50,0.60],[0.10,0.20]$} & 1 & 0.700 & 0.250 & 0.700 & 0.485 & 0.910 & 0.350 & 0.460 & 0.720 & 0.250 & 0.465 & 0.330 & 0.790 \\
\hline A4 & {$[0.18,0.60],[0.10,0.30]$} & 1 & 0.590 & -0.020 & 0.595 & 0.289 & 0.832 & 0.077 & 0.286 & 0.615 & 0.136 & 0.291 & 0.571 & 0.792 \\
\hline B4 & {$[0.28,0.50],[0.00,0.40]$} & 2 & 0.590 & -0.020 & 0.595 & 0.290 & 0.832 & 0.046 & 0.307 & 0.584 & 0.110 & 0.290 & 0.509 & 0.796 \\
\hline A5 & {$[0.50,0.60],[0.10,0.30]$} & 2 & 0.750 & 0.300 & 0.675 & 0.465 & 0.938 & 0.285 & 0.457 & 0.695 & 0.200 & 0.435 & 0.280 & 0.875 \\
\hline B5 & {$[0.60,0.70],[0.05,0.15]$} & 1 & 0.750 & 0.400 & 0.775 & 0.618 & 0.938 & 0.520 & 0.571 & 0.818 & 0.330 & 0.583 & 0.275 & 0.840 \\
\hline A6 & {$[0.35,0.45],[0.20,0.30]$} & 2 & 0.650 & 0.050 & 0.575 & 0.253 & 0.878 & 0.058 & 0.299 & 0.545 & 0.098 & 0.298 & 0.385 & 0.740 \\
\hline B6 & {$[0.30,0.50],[0.15,0.35]$} & 1 & 0.650 & 0.050 & 0.575 & 0.260 & 0.878 & 0.043 & 0.299 & 0.560 & 0.094 & 0.290 & 0.420 & 0.810 \\
\hline A7 & {$[0.00,0.00],[0.10,0.10]$} & 1 & 0.100 & -0.900 & 0.450 & -0.100 & 0.190 & -0.190 & -0.003 & 0.000 & -0.095 & 0.000 & 0.900 & 0.100 \\
\hline$B 7$ & {$[0.00,0.00],[0.90,0.90]$} & 2 & 0.900 & -0.100 & 0.050 & -0.900 & 0.990 & -0.990 & -0.270 & 0.000 & -0.495 & 0.000 & 0.100 & 0.900 \\
\hline$A 8$ & {$[0.30,0.50],[0.30,0.50]$} & 2 & 0.800 & 0.200 & 0.500 & 0.170 & 0.960 & -0.100 & 0.267 & 0.500 & 0.000 & 0.230 & 0.240 & 0.960 \\
\hline$B 8$ & {$[0.40,0.50],[0.40,0.50]$} & 1 & 0.900 & 0.350 & 0.500 & 0.205 & 0.990 & -0.050 & 0.300 & 0.500 & 0.000 & 0.245 & 0.110 & 0.990 \\
\hline A9 & {$[0.40,0.60],[0.00,, 0.30]$} & 2 & 0.650 & 0.150 & 0.675 & 0.440 & 0.878 & 0.260 & 0.413 & 0.700 & 0.208 & 0.410 & 0.425 & 0.835 \\
\hline$B 9$ & {$[0.30,0.70],[0.10,0.20]$} & 1 & 0.650 & 0.150 & 0.675 & 0.435 & 0.878 & 0.285 & 0.397 & 0.725 & 0.243 & 0.415 & 0.475 & 0.815 \\
\hline A10 & {$[0.00,0.00],[0.10,0.30]$} & 2 & 0.200 & -0.800 & 0.400 & -0.200 & 0.360 & -0.370 & -0.010 & 0.000 & -0.170 & 0.000 & 0.870 & 0.280 \\
\hline$B 10$ & {$[0.00,0.00],[0.00,0.20]$} & 1 & 0.100 & -0.900 & 0.450 & -0.100 & 0.190 & -0.200 & 0.000 & 0.000 & -0.090 & 0.000 & 0.980 & 0.180 \\
\hline A11 & {$[0.20,0.40],[0.20,0.40]$} & 2 & 0.600 & -0.100 & 0.500 & 0.100 & 0.840 & -0.140 & 0.200 & 0.440 & 0.000 & 0.200 & 0.480 & 0.760 \\
\hline$B 11$ & {$[0.15,0.45],[0.15,0.45]$} & 1 & 0.600 & -0.100 & 0.500 & 0.113 & 0.840 & -0.165 & 0.200 & 0.465 & 0.000 & 0.188 & 0.520 & 0.810 \\
\hline A12 & {$[0.45,0.55],[0.02,0.18]$} & 2 & 0.600 & 0.100 & 0.700 & 0.454 & 0.840 & 0.350 & 0.415 & 0.707 & 0.263 & 0.446 & 0.448 & 0.712 \\
\hline B12 & {$[0.40,0.60],[0.01,0.19]$} & 1 & 0.600 & 0.100 & 0.700 & 0.459 & 0.840 & 0.343 & 0.413 & 0.719 & 0.263 & 0.441 & 0.478 & 0.754 \\
\hline A13 & {$[0.10,0.20],[0.60,0.80]$} & 2 & 0.850 & 0.000 & 0.225 & -0.440 & 0.978 & -0.670 & -0.053 & 0.180 & -0.290 & 0.040 & 0.165 & 0.975 \\
\hline$B 13$ & {$[0.40,0.50],[0.00,0.10]$} & 1 & 0.500 & -0.050 & 0.700 & 0.425 & 0.750 & 0.370 & 0.367 & 0.680 & 0.290 & 0.425 & 0.550 & 0.590 \\
\hline A14 & {$[0.10,0.20],[0.30,0.40]$} & 2 & 0.500 & -0.350 & 0.400 & -0.145 & 0.750 & -0.380 & 0.067 & 0.230 & -0.145 & 0.095 & 0.550 & 0.590 \\
\hline B14 & {$[0.00,0.30],[0.20,0.50]$} & 1 & 0.500 & -0.350 & 0.400 & -0.125 & 0.750 & -0.420 & 0.067 & 0.270 & -0.135 & 0.075 & 0.650 & 0.710 \\
\hline A15 & {$[0.13,0.26],[0.17,0.43]$} & 1 & 0.495 & -0.310 & 0.448 & -0.038 & 0.745 & -0.282 & 0.117 & 0.306 & -0.074 & 0.128 & 0.591 & 0.648 \\
\hline B15 & {$[0.17,0.22],[0.09,0.51]$} & 2 & 0.495 & -0.310 & 0.448 & -0.041 & 0.745 & -0.306 & 0.127 & 0.299 & -0.070 & 0.131 & 0.580 & 0.641 \\
\hline A16 & {$[0.20,0.20],[0.40,0.80]$} & 2 & 0.800 & 0.000 & 0.300 & -0.280 & 0.960 & -0.560 & 0.040 & 0.240 & -0.200 & 0.080 & 0.200 & 0.920 \\
\hline B16 & {$[0.20,0.20],[0.20,0.35]$} & 1 & 0.475 & -0.325 & 0.463 & -0.020 & 0.724 & -0.225 & 0.123 & 0.305 & -0.054 & 0.145 & 0.559 & 0.539 \\
\hline A17 & {$[0.20,0.30],[0.50,0.60]$} & 2 & 0.800 & 0.050 & 0.350 & -0.160 & 0.960 & -0.415 & 0.087 & 0.305 & -0.173 & 0.110 & 0.220 & 0.890 \\
\hline$B 17$ & {$[0.30,0.40],[0.20,0.40]$} & 1 & 0.650 & 0.000 & 0.525 & 0.160 & 0.878 & -0.070 & 0.247 & 0.480 & 0.030 & 0.240 & 0.395 & 0.775 \\
\hline A18 & {$[0.20,0.40],[0.20,0.30]$} & 2 & 0.550 & -0.150 & 0.525 & 0.130 & 0.798 & -0.070 & 0.207 & 0.450 & 0.038 & 0.220 & 0.525 & 0.675 \\
\hline B18 & {$[0.30,0.50],[0.10,0.20]$} & 1 & 0.550 & -0.050 & 0.625 & 0.315 & 0.798 & 0.175 & 0.310 & 0.595 & 0.178 & 0.335 & 0.525 & 0.675 \\
\hline A19 & {$[0.60,0.70],[0.20,0.30]$} & 2 & 0.900 & 0.550 & 0.700 & 0.565 & 0.990 & 0.370 & 0.553 & 0.720 & 0.210 & 0.485 & 0.110 & 0.990 \\
\hline B19 & {$[0.50,0.70],[0.10,0.20]$} & 1 & 0.750 & 0.350 & 0.725 & 0.545 & 0.938 & 0.405 & 0.510 & 0.765 & 0.273 & 0.505 & 0.295 & 0.875 \\
\hline$A 20$ & {$[0.50,0.50],[0.063,0.25]$} & 1 & 0.657 & 0.157 & 0.672 & 0.422 & 0.882 & 0.281 & 0.411 & 0.672 & 0.215 & 0.422 & 0.367 & 0.733 \\
\hline
\end{tabular}


Melda KOKOC, Suleyman ERSOZ/ GUJ Sci, 35(4): 1484-1502 (2022)

$\begin{array}{lllll}B 20 & {[0.50,0.50],[0.031,0.28]} & 2 & \mathbf{0 . 6 5 7} & \mathbf{0 . 1 5 7}\end{array}$

0.672

0.422

$\mathbf{0 . 8 8 2}$

0.274

0.414

$\mathbf{0 . 6 7 2} \quad 0.209$

0.422 
Table 5. Decision matrix $R_{4 \times 5}$

\begin{tabular}{cccccc}
\hline & $\mathrm{C} 1$ & $\mathrm{C} 2$ & $\mathrm{C} 3$ & $\mathrm{C} 4$ & $\mathrm{C} 5$ \\
\hline \multirow{2}{*}{$\mathrm{A} 1$} & {$[0.4,0.5]$,} & {$[0.5,0.6]$,} & {$[0.3,0.4]$,} & {$[0.7,0.8]$,} & {$[0.5,0.6]$,} \\
& {$[0.1,0.3]$} & {$[0.1,0.2]$} & {$[0.2,0.3]$} & {$[0.1,0.2]$} & {$[0.1,0.2]$} \\
$\mathrm{A} 2$ & {$[0.5,0.6]$,} & {$[0.3,0.4]$,} & {$[0.7,0.8]$,} & {$[0.3,0.4]$,} & {$[0.4,0.5]$,} \\
& {$[0.1,0.2]$} & {$[0.1,0.3]$} & {$[0.1,0.2]$} & {$[0.3,0.4]$} & {$[0.1,0.2]$} \\
$\mathrm{A} 3$ & {$[0.6,0.7]$,} & {$[0.7,0.8]$,} & {$[0.5,0.6]$,} & {$[0.4,0.5]$,} & {$[0.3,0.5]$,} \\
& {$[0.1,0.2]$} & {$[0.1,0.2]$} & {$[0.3,0.4]$} & {$[0.3,0.4]$} & {$[0.3,0.4]$} \\
& {$[0.5,0.6]$,} & {$[0.4,0.5]$,} & {$[0.6,0.7]$,} & {$[0.6,0.7]$,} & {$[0.6,0.7]$,} \\
A4 & {$[0.2,0.3]$} & {$[0.3,0.4]$} & {$[0.2,0.3]$} & {$[0.2,0.3]$} & {$[0.1,0.3]$} \\
\hline
\end{tabular}

The aggregated IVIF value $\gamma_{i}$ for each alternatives $A_{i}$ is obtained using IVIFWA operator as $\gamma_{1}=[0.48$, $0.48],[0.11,0.23], \gamma_{2}=[0.37,0.47],[0.11,0.24], \gamma_{3}=[0.50,0.61],[0.17,0.28], \gamma_{4}=[0,41,0.50],[0.19$, $0.33]$. Then ranking values are calculated as $\operatorname{MK}\left(\mathrm{A}_{1}\right)=0.15, \operatorname{MK}\left(\mathrm{A}_{2}\right)=0.08, \operatorname{MK}\left(\mathrm{A}_{3}\right)=0.25, \operatorname{MK}\left(\mathrm{A}_{4}\right)=0.09$ by using score function $\mathrm{MK}\left(\right.$.). Thus, the rank of the alternatives is obtained as $A_{3}>A_{1}>A_{4}>A_{2}$.

Example 21. [25] The performance of four teachers (A1, A2, A3, A4) are evaluated on the basis the five criteria: (C1) subject command of the teacher, (C2) ability to communicate, (C3) ability to create interest in the course, (C4) ability to ensure course coverage, (C5) ability to give suitable illustrations and to create linkage. The criteria' weights are $w=[0.1,0.2,0.25,0.3,0.15]^{T}$. The decision matrix $D_{4 \times 5}$ (Table 6) includes information related to the evaluation of teachers on criteria.

Table 6. Decision matrix $D_{4 \times 5}$

\begin{tabular}{llllll}
\hline & $\mathrm{C} 1$ & $\mathrm{C} 2$ & $\mathrm{C} 3$ & $\mathrm{C} 4$ & $\mathrm{C} 5$ \\
\hline \multirow{2}{*}{ A1 } & {$[0.16,0.16]$,} & {$[0.24,0.76]$,} & {$[0.00,0.00]$,} & {$[0.24,0.24]$,} & {$[0,00,0.00]$,} \\
& {$[0.17,0.50]$} & {$[0,00,0.00]$} & {$[0.50,0.50]$} & {$[0.26,0.26]$} & {$[0.25,0.75]$} \\
$\mathrm{A} 2$ & {$[0.16,0.16]$,} & {$[0.14,0.45]$,} & {$[0.24,0.24]$,} & {$[0.21,0.21]$,} & {$[0.16,0.16]$,} \\
& {$[0.17,0.50]$} & {$[0.15,0.25]$} & {$[0.26,0.26]$} & {$[0.22,0.36]$} & {$[0.17,0.50]$} \\
& {$[0.24,0.24]$,} & {$[0.24,0.24]$,} & {$[0.24,0.24]$,} & {$[0.00,0.00]$,} & {$[0.00,0.00]$,} \\
A3 & {$[0.26,0.26]$} & {$[0.26,0.26]$} & {$[0.26,0.26]$} & {$[0.50,0.50]$} & {$[0.25,0.75]$} \\
& {$[0.10,0.30]$,} & {$[0.13,0.38]$,} & {$[0.12,0.38]$,} & {$[0.16,0.50]$,} & {$[0.00,0.00]$,} \\
A4 & {$[0.30,0.30]$} & {$[0.13,0.38]$} & {$[0.13,0.38]$} & {$[0.17,0.17]$} & {$[0.25,0.75]$} \\
& & & &
\end{tabular}

The aggregated IVIF value $\gamma_{i}$ for each alternative $A_{i}$ is calculated using IVIFWA operator as $\gamma_{1}$ $=[0.14,0.32],[0,0], \gamma_{2}=[0.17,0.24],[0.20,0.33], \gamma_{3}=[0.14,0.14],[0.31,0.37], \gamma_{4}=[0.11,0.37],[0.17$, 0.32]. Then ranking values are calculated as $\operatorname{MK}\left(\mathrm{A}_{1}\right)=-0.11, \operatorname{MK}\left(\mathrm{A}_{2}\right)=-0.25, \operatorname{MK}\left(\mathrm{A}_{3}\right)=-0.37, \operatorname{MK}\left(\mathrm{A}_{4}\right)=-$ 0.16 by using score function $\mathrm{MK}\left(\right.$.). Thus, the rank of the alternatives is obtained as $A_{1}>A_{4}>A_{2}>A_{3}$.

To validate the applicability and feasibility of the proposed new approach, a comparative study with existing ranking functions is conducted on the basis of Example 20 and Example 21. The ranking results obtained from the existing ranking functions are listed in Table 7 for both examples. Table 7 shows that the ranking results derived from the score functions in [10, 13, 16, 19] and accuracy functions in [7] are identical to the ranking results $\left(A_{3}>A_{1}>A_{4}>A_{2}\right.$ ) of the new score function for Example 20. In addition, the ranking of the first two alternatives $\left(A_{3}>A_{1}\right)$ obtained from the score functions in $[9,12,14,17]$ and accuracy functions in [20-23, 25] are the same as the rank obtained from the proposed function. It is seen from Table 7 that ranking results obtained from the score functions in $[6,9,10,12,14,15,17-19]$ and accuracy functions in [7, 20-25] are identical to the ranking results $\left(A_{1}>A_{4}>A_{2}>A_{3}\right)$ of the proposed new score function for Example 21. 
Table 7. Ranking results obtained from different functions

\begin{tabular}{|c|c|c|c|c|}
\hline References & \multicolumn{2}{|l|}{ Example 20} & \multicolumn{2}{|l|}{ Example 21} \\
\hline \multirow{2}{*}{$\mathrm{Xu}[12]$} & $S\left(A_{1}\right)=0.31, S\left(A_{2}\right)=0.24, S\left(A_{3}\right)=0.33, S\left(A_{4}\right)=0.19$ & $A_{3}>A_{1}>A_{2}>A_{4}$ & $S\left(A_{1}\right)=0.23, S\left(A_{2}\right)=-0.06, S\left(A_{3}\right)=-0.20, S\left(A_{4}\right)=0.00$ & $A_{1}>A_{4}>A_{2}>A_{3}$ \\
\hline & $H\left(A_{1}\right)=0.65, H\left(A_{2}\right)=0.60, H\left(A_{3}\right)=0.78, H\left(A_{4}\right)=0.71$ & $A_{3}>A_{4}>A_{1}>A_{2}$ & $H\left(A_{1}\right)=0.23, H\left(A_{2}\right)=0.47, H\left(A_{3}\right)=0.483, H\left(A_{4}\right)=0.484$ & $A_{4}>A_{3}>A_{2}>A_{1}$ \\
\hline Lee [13] & $S\left(A_{1}\right)=1.54, S\left(A_{2}\right)=1.37, S\left(A_{3}\right)=1.85, S\left(A_{4}\right)=1.51$ & $A_{3}>A_{1}>A_{4}>A_{2}$ & $S\left(A_{1}\right)=0.97, S\left(A_{2}\right)=0.91, S\left(A_{3}\right)=0.78, S\left(A_{4}\right)=0.98$ & $A_{4}>A_{1}>A_{2}>A_{3}$ \\
\hline Ye [5] & $M\left(A_{1}\right)=0.13, M\left(A_{2}\right)=0.01, M\left(A_{3}\right)=0.34, M\left(A_{4}\right)=0.16$ & $A_{3}>A_{4}>A_{1}>A_{2}$ & $M\left(A_{1}\right)=-0.54, M\left(A_{2}\right)=-0.32, M\left(A_{3}\right)=-0.38, M\left(A_{4}\right)=-0.28$ & $A_{4}>A_{2}>A_{3}>A_{1}$ \\
\hline Nayagam et al. [20] & $L\left(A_{1}\right)=0.39, L\left(A_{2}\right)=0.32, L\left(A_{3}\right)=0.46, L\left(A_{4}\right)=0.31$ & $A_{3}>A_{1}>A_{2}>A_{4}$ & $L\left(A_{1}\right)=0.23, L\left(A_{2}\right)=0.00, L\left(A_{3}\right)=-0.15, L\left(A_{4}\right)=0.06$ & $A_{1}>A_{4}>A_{2}>A_{3}$ \\
\hline $\begin{array}{l}\text { Nayagam and } \\
\text { Sivaraman }[21]\end{array}$ & $L G\left(A_{1}\right)=0.65, L G\left(A_{2}\right)=0.62, L G\left(A_{3}\right)=0.66, L G\left(A_{4}\right)=0.60$ & $A_{3}>A_{1}>A_{2}>A_{4}$ & $L G\left(A_{1}\right)=0.62, L G\left(A_{2}\right)=0.47, L G\left(A_{3}\right)=0.40, L G\left(A_{4}\right)=0.50$ & $A_{1}>A_{4}>A_{2}>A_{3}$ \\
\hline Bai [14] & $I\left(A_{1}\right)=0.65, I\left(A_{2}\right)=0.582, I\left(A_{3}\right)=0.67, I\left(A_{4}\right)=0.578$ & $A_{3}>A_{1}>A_{2}>A_{4}$ & $I\left(A_{1}\right)=0.40, I\left(A_{2}\right)=0.31, I\left(A_{3}\right)=0.21, I\left(A_{4}\right)=0.34$ & $A_{1}>A_{4}>A_{2}>A_{3}$ \\
\hline \multirow{2}{*}{ Wang and Niu [15] } & $W_{S}\left(A_{1}\right)=0.42, W_{S}\left(A_{2}\right)=0.34, W_{S}\left(A_{3}\right)=0.40, W_{S}\left(A_{4}\right)=0.25$ & $A_{1}>A_{3}>A_{2}>A_{4}$ & $W_{S}\left(A_{1}\right)=0.41, W_{S}\left(A_{2}\right)=-0.09, W_{S}\left(A_{3}\right)=-0.31, W_{S}\left(A_{4}\right)=-0.01$ & $A_{1}>A_{4}>A_{2}>A_{3}$ \\
\hline & $W_{H}\left(A_{1}\right)=0.88, W_{H}\left(A_{2}\right)=0.84, W_{H}\left(A_{3}\right)=0.95, W_{H}\left(A_{4}\right)=0.92$ & $A_{3}>A_{4}>A_{1}>A_{2}$ & $W_{H}\left(A_{1}\right)=0.41, W_{H}\left(A_{2}\right)=0.72, W_{H}\left(A_{3}\right)=0.732, W_{H}\left(A_{4}\right)=0.734$ & $A_{4}>A_{3}>A_{2}>A_{1}$ \\
\hline $\begin{array}{l}\text { Joshi and Kharayat } \\
\text { [7] }\end{array}$ & $P\left(A_{1}\right)=0.39, P\left(A_{2}\right)=0.33, P\left(A_{3}\right)=0.45, P\left(A_{4}\right)=0.35$ & $A_{3}>A_{1}>A_{4}>A_{2}$ & $P\left(A_{1}\right)=0.17, P\left(A_{2}\right)=0.13, P\left(A_{3}\right)=0.06, P\left(A_{4}\right)=0.16$ & $A_{1}>A_{4}>A_{2}>A_{3}$ \\
\hline Kang et al. [22] & $H_{K}\left(A_{1}\right)=0.25, H_{K}\left(A_{2}\right)=0.16, H_{K}\left(A_{3}\right)=0.27, H_{K}\left(A_{4}\right)=0.11$ & $A_{3}>A_{1}>A_{2}>A_{4}$ & $H_{K}\left(A_{1}\right)=0.23, H_{K}\left(A_{2}\right)=-0.21, H_{K}\left(A_{3}\right)=0.38, H_{K}\left(A_{4}\right)=-0.15$ & $A_{1}>A_{4}>A_{2}>A_{3}$ \\
\hline Zhang and $\mathrm{Xu}[24]$ & $F\left(A_{1}\right)=0.20, F\left(A_{2}\right)=0.16, F\left(A_{3}\right)=0.19, F\left(A_{4}\right)=0.12$ & $A_{1}>A_{3}>A_{2}>A_{4}$ & $F\left(A_{1}\right)=0.21, F\left(A_{2}\right)=-0.05, F\left(A_{3}\right)=-0.15, F\left(A_{4}\right)=0.00$ & $A_{1}>A_{4}>A_{2}>A_{3}$ \\
\hline $\begin{array}{l}\text { Joshi and Kumar } \\
\text { [25] }\end{array}$ & $T\left(A_{1}\right)=0.40, T\left(A_{2}\right)=0.34, T\left(A_{3}\right)=0.43, T\left(A_{4}\right)=0.33$ & $A_{3}>A_{1}>A_{2}>A_{4}$ & $T\left(A_{1}\right)=0.23, T\left(A_{2}\right)=0.15, T\left(A_{3}\right)=0.09, T\left(A_{4}\right)=0.17$ & $A_{1}>A_{4}>A_{2}>A_{3}$ \\
\hline \multirow{2}{*}{$\begin{array}{l}\text { Wang and Chen } \\
\text { [17] }\end{array}$} & $S_{N W C}\left(A_{1}\right)=0.16, S_{N W C}\left(A_{2}\right)=0.08, S_{N W C}\left(A_{3}\right)=0.17, S_{N W C}\left(A_{4}\right)=0.06$ & $A_{3}>A_{1}>A_{2}>A_{4}$ & $S_{N W C}\left(A_{1}\right)=0.03, S_{N W C}\left(A_{2}\right)=-0.08, S_{N W C}\left(A_{3}\right)=-0.11, S_{N W C}\left(A_{4}\right)=-0.10$ & $A_{1}>A_{2}>A_{4}>A_{3}$ \\
\hline & $H_{N W C}\left(A_{1}\right)=0.37, H_{N W C}\left(A_{2}\right)=0.45, H_{N W C}\left(A_{3}\right)=0.24, H_{N W C}\left(A_{4}\right)=0.32$ & $A_{2}>A_{1}>A_{4}>A_{3}$ & $H_{N W C}\left(A_{1}\right)=0.84, H_{N W C}\left(A_{2}\right)=0.58, H_{N W C}\left(A_{3}\right)=0.53, H_{N W C}\left(A_{4}\right)=0.63$ & $A_{1}>A_{4}>A_{2}>A_{3}$ \\
\hline \multirow{2}{*}{ Gong and $\mathrm{Ma}[18]$} & $S_{G M}\left(A_{1}\right)=0.74, S_{G M}\left(A_{2}\right)=0.706, S_{G M}\left(A_{3}\right)=0.709, S_{G M}\left(A_{4}\right)=0.64$ & $A_{1}>A_{3}>A_{2}>A_{4}$ & $S_{G M}\left(A_{1}\right)=0.97, S_{G M}\left(A_{2}\right)=0.44, S_{G M}\left(A_{3}\right)=0.29, S_{G M}\left(A_{4}\right)=0.47$ & $A_{1}>A_{4}>A_{2}>A_{3}$ \\
\hline & $H_{G M}\left(A_{1}\right)=0.702, H_{G M}\left(A_{2}\right)=0.696, H_{G M}\left(A_{3}\right)=0.88, H_{G M}\left(A_{4}\right)=0.81$ & $A_{3}>A_{4}>A_{1}>A_{2}$ & $H_{G M}\left(A_{1}\right)=0.30, H_{G M}\left(A_{2}\right)=0.56, H_{G M}\left(A_{3}\right)=0.51, H_{G M}\left(A_{4}\right)=0.64$ & $A_{4}>A_{2}>A_{3}>A_{1}$ \\
\hline Jia, et al. [19] & $J\left(A_{1}\right)=0.08, J\left(A_{2}\right)=0.06, J\left(A_{3}\right)=0.10, J\left(A_{4}\right)=0.05$ & $A_{3}>A_{1}>A_{4}>A_{2}$ & $J\left(A_{1}\right)=0.03, J\left(A_{2}\right)=-0.01, J\left(A_{3}\right)=-0.04, J\left(A_{4}\right)=0.00$ & $A_{1}>A_{4}>A_{2}>A_{3}$ \\
\hline This study & $M K\left(A_{1}\right)=0.15, M K\left(A_{2}\right)=0.08, M K\left(A_{3}\right)=0.25, M K\left(A_{4}\right)=0.09$ & $A_{3}>A_{1}>A_{4}>A_{2}$ & $M K\left(A_{1}\right)=-0.11, M K\left(A_{2}\right)=-0.25, M K\left(A_{3}\right)=-0.37, M K\left(A_{4}\right)=-0.16$ & $A_{1}>A_{4}>A_{2}>A_{3}$ \\
\hline
\end{tabular}


It is remarkable that the ranking functions of the IVIFVs affect the ranking results, and so, ranking orders may be obtained differently with different functions. For this reason, in decision-making problems, it is necessary to use ranking functions that have the monotonicity property and do not have drawbacks in the ranking of the IVIFVs. Otherwise, misleading results may be produced for the decision-maker. The proposed new score function has the monotonicity property. It also copes with the shortcomings of the existing functions. Thus, the new score function may present an acceptable rank for IVIFVs, and it can be used effectively for decision-making problems.

\section{CONCLUSION}

The ranking of IVIFVs is one of the most popular fields in several real-world decision-making problems. It is well recognized that many researchers have been working on the ranking of IVIFVs. As far as we know, there is no general approach that ranks any two IVIFVs. Many researchers have suggested numerous score functions and accuracy functions. But it is pointed out that in some cases, these mentioned functions are not always effective. In this study, a new score function is developed and a ranking method based on this score function is introduced under the IVIF environment. The main contributions of this study are presented:

(i) It is shown that both the most popular ranking functions and their improved versions may produce counter-intuitive ranking results.

(ii) A new score function which can rank any two IVIFVs is developed. This function has monotonicity proper-ty, and so, score value increases with the increasing of $\mu^{L}, \mu^{U}$ whereas it decreases with the increasing of $v^{L}, v^{U}$. Furthermore, it is noteworthy that the new function overcomes the shortcomings of the other ranking functions.

(iii) Decision-making process has ambiguity or vagueness. The proposed decision algorithm is suitable for ambiguity or vagueness environments.

(iv) Two illustrative examples are given to show the applicability and effectiveness of the proposed approach.

\section{CONFLICTS OF INTEREST}

No conflict of interest was declared by the authors.

\section{REFERENCES}

[1] Wei, A. P., Li, D. F., Lin, P. P., Jiang, B. Q., "An information-based score function of interval-valued intuitionistic fuzzy sets and its application in multiattribute decision making", Soft Computing, 1-11, (2020).

[2] Zadeh, L. A., "Fuzzy sets", Information Control, 8(3): 338-353, (1965).

[3] Atanassov, K., "Intuitionistic fuzzy sets", Fuzzy Sets and Systems, 20(1): 87-96, (1986).

[4] Niu, L. L., Li, J., Li, F. L., Wang, Z. X., "Multi-criteria decision-making method with double risk parameters in interval-valued intuitionistic fuzzy environments", Complex \& Intelligent Systems, 6(3): 669-679, (2020).

[5] Ye, J., "Multicriteria fuzzy decision-making method based on a novel accuracy function under interval-valued intuitionistic fuzzy environment", Expert Systems with Applications, 36(3): 68996902, (2009).

[6] Tu, C. C., Chen, L. H., "Novel score functions for interval-valued intuitionistic fuzzy values", Proceedings of SICE Annual Conference (SICE), (2012). 
[7] Joshi, B. P., Kharayat, P. S., "An accuracy function for interval-valued intuitionistic fuzzy number", International Journal of Mathematical Archive, 6(1): 51-55, (2015).

[8] Atanassov, K., Gargov, G., "Interval valued intuitionistic fuzzy sets", Fuzzy Sets and Systems, 31: 343-349, (1989).

[9] Garg, H., "A new generalized improved score function of interval-valued intuitionistic fuzzy sets and applications in expert systems", Applied Soft Computing, 38: 988-999, (2016).

[10] Nayagam, V. L. G., Jeevaraj, S., Dhanasekaran, P., "An intuitionistic fuzzy multi-criteria decisionmaking method based on non-hesitance score for interval-valued intuitionistic fuzzy sets", Soft Computing, 21(23): 7077-7082, (2017).

[11] Talukdar, P., Dutta, P., "A new ranking approach for interval valued intuitionistic fuzzy sets and its application in decision making", International Journal of Fuzzy System Applications, 8(2): 89-104, (2019).

[12] $\mathrm{Xu}, \mathrm{Z}$. S., "Methods for aggregating interval-valued intuitionistic fuzzy information and their application to decision making", Control and Decision, 22(2): 215-219, (2007).

[13] Lee, W., "A novel method for ranking interval-valued intuitionistic fuzzy numbers and its application to decision making", International Conference on Intelligent Human-Machine Systems and Cybernetics, (2009).

[14] Bai, Z. Y., "An interval-valued intuitionistic fuzzy TOPSIS method based on an improved score function", Scientific World Journal, 879089, (2013).

[15] Wang, Z. X., Niu, L. L., "A new scoring function of intervai-valued intuitionistic fuzzy number and its application in multi-attribute decision making", Fuzzy Systems and Mathematics, 27(4): 167-172, (2013).

[16] Wang, C. Y., Chen, S. M., "An improved multiattribute decision making method based on new score function of interval-valued intuitionistic fuzzy values and linear programming methodology", Information Sciences, 411: 176-184, (2017).

[17] Wang, C. Y., Chen, S. M., "A new multiple attribute decision making method based on linear programming methodology and novel score function and novel accuracy function of interval-valued intuitionistic fuzzy values", Information Sciences, 438: 145-155, (2018).

[18] Gong, R., Ma, L., "A new score function and accuracy function of interval-valued intuitionistic fuzzy number and its application", Systems Engineering -Theory \& Practice, 30(2): 463- 475, (2019).

[19] Jia, Q., Hu, J., Zhang, W., "A novel fault detection model based on Atanassov's interval-valued intuitionistic fuzzy sets", Belief Rule Base and Evidential Reasoning, IEEE Access, 8: 4551-4567, (2019).

[20] Nayagam, V. L. G., Muralikrishnan, S., Sivaraman, G., "Multi-criteria decision-making method based on interval-valued intuitionistic fuzzy sets", Expert Systems with Applications, 38(3), 14641467, (2011).

[21] Nayagam, V. L. G., Sivaraman, G., "Ranking of interval-valued intuitionistic fuzzy sets", Applied Soft Computing, 11(4): 3368-3372, (2011). 
[22] Kang, J., Lan, R., Wang, S. S., "A new accuracy function of interval-valued intuitionnistic fuzzy numbers and its application in decision making", Journal of Xi'an Uniandrsity of Post and Telecommunications, 20(3): 86-91, (2015).

[23] Sahin, R., "Fuzzy multicriteria decision making method based on the improved accuracy function for interval-valued intuitionistic fuzzy sets", Soft Computing, 20(7): 2557-2563, (2016).

[24] Zhang, F., Xu, S., "Remarks to "Fuzzy multicriteria decision making method based on the improved accuracy function for interval-valued intuitionistic fuzzy sets", Soft Computing, 21(9): 2263-2268, (2017).

[25] Joshi, D., Kumar, S., "Improved accuracy function for interval-valued intuitionistic fuzzy sets and its application to multi-attributes group decision making", Cybernetics and Systems, 49(1): 64-76, (2018).

[26] Huang, W. W., Zhang, F. W., Xu, S. H., "A complete ranking method for interval-valued intuitionistic fuzzy numbers and its applications to multicriteria decision making", Soft Computing, $1-8,(2020)$.

[27] Nguyen, H., "A generalized p-norm knowledge-based score function for an interval-valued intuitionistic fuzzy set in decision making", IEEE Transactions on Fuzzy Systems, 28(3): 409-423, (2019).

[28] Hao, Y., Chen, X., "Study on the ranking problems in multiple attribute decision making based on interval-valued intuitionistic fuzzy numbers", International Journal of Intelligent Systems, 33(3): 560-572, (2018).

[29] Liu, B., Luo, M. X., "Multicriteria decision making based on interval-valued intuitionistic fuzzy sets with a new kind of accuracy function", Quantitative Logic and Soft Computing 2016: Springer, 477486, (2017). 